This is an Open Access article licensed under the terms of the Creative Commons AttributionNonCommercial-NoDerivs 3.0 License (www.karger.com/OA-license), applicable to the online version of the article only. Distribution for non-commercial purposes only.

\title{
Recommended Measures for the Assessment of Cognitive and Physical Performance in Older Patients with Dementia: A Systematic Review
}

\author{
Willem J.R. Bossers ${ }^{a}$ Lucas H.V. van der Woude ${ }^{a, b}$ Froukje Boersma ${ }^{c}$ \\ Erik J.A. Scherder ${ }^{a}$ d Marieke J.G. van Heuvelen ${ }^{a}$ \\ ${ }^{a}$ Center for Human Movement Sciences, ${ }^{b}$ Center for Rehabilitation, and ${ }^{c}$ Department of General Practice, \\ Elderly Care Medicine, University of Groningen, University Medical Center Groningen, Groningen, and \\ ${ }^{\mathrm{d}}$ Department of Clinical Neuropsychology, VU University, Amsterdam, The Netherlands
}

\section{Key Words}

Dementia $\cdot$ Neuropsychological tests $\cdot$ Exercise tests $\cdot$ Tool use $\cdot$ Outcome measures

Systematic review

\begin{abstract}
Aim/Goal: To recommend a set of neuropsychological and physical exercise tests for researchers to assess cognition and physical fitness in clinical trials with older patients with dementia; to create consensus, decrease heterogeneity, and improve research quality. Methods: A literature search (2005-2011) yielded 89 randomized controlled trials. To provide information on test recommendations the frequency of test use, effect size of the test outcome, study quality, and psychometric properties of tests were analyzed. Results: Fifty-nine neuropsychological tests (cognitive domains: global cognition, executive functioning, memory, and attention) and 10 exercise tests (physical domains: endurance capacity, muscle strength, balance, and mobility) were found. Conclusion: The Severe Impairment Battery, Mini Mental State Examination, and Alzheimer Disease Assessment Scale - cognitive subscale were recommended to measure global cognition. The Verbal Fluency Test Category/Letters, Clock Drawing Test, and Trail Making Test-B were recommended to measure executive functioning. No specific memory test could be recommended. The Digit Span Forward, Digit Span Backward, and Trail Making Test-A were recommended to measure attention. As physical exercise tests, the Timed Up and Go and Six Meter Walk for mobility, the Six Minute Walk Distance for endurance capacity, and the Tinetti Balance Scale were recommended.




\section{Introduction}

With the worldwide trend of an aging population, the number of patients with dementia will increase dramatically in the coming decades [1]. Dementia is characterized by a loss of neurons and atrophy of brain tissue [2-5]. Eventually, this leads to limitations in cognitive performance of executive functioning, memory, and attention $[6,7]$. The neurodegenerative processes in the brain go hand in hand with limitations in physical performance of endurance capacity, muscle strength, balance, and mobility $[6,8]$. Eventually, decline in cognitive performance and physical performance results in problems in activities of daily living and behavior, leading to institutionalization and a decreased quality of life $[3,9,10]$. Therefore, prevention of decline and preferably an improvement in both cognitive and physical performance in patients with dementia are of utmost importance.

With the growing impact of dementia on today's society, new treatments need to be developed that effectively reduce the limitations caused by a decline of cognitive and physical performance in patients with dementia [11]. Meta-analysis and systematic reviews reported that pharmacological (e.g. medication) and non-pharmacological (e.g. exercise) interventions may have a positive effect on cognition and physical functioning [12-18]. However, the individual studies in these reviews showed ambiguous results and the tests that measured cognitive and physical functioning appeared to show large heterogeneity. Consequently, the comparability of the outcomes of clinical trials is hampered [19]. Therefore, future intervention studies that aim to improve cognitive functioning, physical functioning, or a combination of both should strive to use a limited number of generally accepted, feasible, reliable, and valid tests that adequately cover the domains of cognitive and physical functioning in patients with dementia. This study is intended to contribute to this goal.

Recommendations on cognitive assessment tests for the purpose of diagnosing dementia were recently provided by Chaves et al. [20] and Young et al. [21]. Information regarding the tests on cognition that researchers should use for measuring treatment effects, however, is not available yet. To the authors' knowledge, recommendations regarding the use of exercise tests evaluating physical functioning in clinical trials are fully lacking.

The aim of this systematic review is to give up-to-date recommendations of both neuropsychological and physical exercise tests for researchers who have the aim to investigate treatment effects on cognition and physical functioning in older patients with dementia. Firstly, a comprehensive overview of tests on the basis of randomized controlled trials (RCTs) is presented. Frequently used neuropsychological and physical exercise tests are evaluated in relation with study quality of RCTs, nature of the interventions in RCTs, type of dementia that was studied, and sensitivity to change of the tests. Secondly, the reliability and validity of frequently used tests was reviewed.

\section{Methods}

\section{Data Sources}

Between August 2010 and August 2011, computer databases PubMed, EMBASE, Biological abstracts, Web of Science (ISI), PsycINFO, CINAHL, and Cochrane Library were searched for relevant studies published between 2005 and 2011. Limits for the searches in the computer databases were set on: clinical trial, humans, and age $\geq 65$ years. Keywords in the search included terms from Medical Subject Headings (MESH) and EMBASE thesaurus (EMTREE). The following terms were used in the MESH database and EMTREE thesaurus: dementia, Alzheimer disease, vascular dementia, frontotemporal dementia, Lewy body disease, neuropsychological tests, and exercise tests. Keywords for dementia 
(dementia OR Alzheimer disease OR vascular dementia OR frontotemporal dementia OR Lewy body disease) were combined (with 'AND') with terms that expressed the use of neuropsychological or exercise tests (neuropsychological tests OR exercise tests). In addition, reference lists of reviews regarding the subject were thoroughly hand searched for additional studies.

\section{Inclusion Criteria}

Studies were included if they met the following criteria: (1) the design was a RCT; (2) the participants had a diagnosis of dementia; (3) the participants were on average older than 65 years; (4) neuropsychological tests and/or exercise tests were used to measure the effects of an intervention, and (5) the study was written in English, German, French or Dutch.

\section{Selection Process}

After the literature search, a first selection of studies was made according to their titles, followed by a selection after reading the abstracts. Two reviewers ( $\mathrm{WB}$ and $\mathrm{MvH}$ ) independently performed both steps to identify those studies that met the inclusion criteria (agreement 94\%, disagreement 6\%). Disagreement was solved with full-text screening. Full-text analysis to check the inclusion criteria was performed for the studies identified in the preceding steps. Subsequently, reviews were hand searched for clinical trials that were not already found in the literature search. Finally, full-text analysis of and data extraction from the selected studies was performed.

\section{Data Extraction}

From the selected RCTs the following data were extracted: neuropsychological tests, physical exercise tests, type of dementia, sample size, and data regarding the intervention description (e.g. pharmacological, exercise). For each neuropsychological or physical exercise test the overall means and standard deviations were calculated from all RCTs that used a given test. Further, on the basis of the selected RCTs the overall means and standard deviations were calculated for age, baseline scores, and posttest scores.

\section{Effect Size}

In order to express the sensitivity to change for each neuropsychological or physical exercise test, Cohen's $d$ effect sizes (ESs) for a test were calculated on the basis of the selected RCTs $[22,23]$. If the mean and standard deviation of pretest and posttest were presented in the RCT, the following formula was used:

$$
d=\left[\left(\text { post }_{\exp }-\text { pre }_{\exp }\right)-\left(\text { post }_{\text {cont }}-\operatorname{pre}_{\text {cont }}\right)\right] / S q r t\left[\left(\left[s^{2} \operatorname{pre}_{\exp }\left(\mathrm{n}_{\exp }\right)+s^{2} \operatorname{pre}_{\text {cont }}\left(\mathrm{n}_{\text {cont }}\right)\right] /\left[\mathrm{n}_{\exp }+\mathrm{n}_{\text {cont }}\right]\right)+\right.
$$
$\left(\left[s^{2}\right.\right.$ post $_{\text {exp }}\left(\mathrm{n}_{\text {exp }}\right)+s^{2}$ post $\left.\left.\left._{\text {cont }}\left(\mathrm{n}_{\text {cont }}\right)\right] /\left[\mathrm{n}_{\text {exp }}+\mathrm{n}_{\text {cont }}\right]\right) / 2\right][24]$

If the means and standard deviations were not presented in the RCT, the $F$ statistic was used with the following formula:

$$
d=\operatorname{Sqrt}\left[F\left(\left[\left(\mathrm{n}_{\exp }+\mathrm{n}_{\text {cont }}\right) /\left(\mathrm{n}_{\exp } \cdot \mathrm{n}_{\text {cont }}\right)\right] \cdot\left[\left(\mathrm{n}_{\exp }+\mathrm{n}_{\text {cont }}\right) /\left(\mathrm{n}_{\exp }+\mathrm{n}_{\text {cont }}-2\right)\right]\right)\right][24]
$$

The overall ES was calculated as the mean of individual ESs weighted for the sample size. Cohen's benchmarks were used to indicate small $(d=0.20)$, medium $(d=0.50)$, and large $(d=0.80)$ ESs [22].

\section{Study Quality}

Study quality of each RCT that used a given test was assessed with the Physiotherapy Evidence Database (PEDro) [25]. According to the PEDro scoring system, a score of 9-10 was considered as excellent, a score of $6-8$ as good, a score of $4-5$ as moderate, and a score of $0-3$ 
Performance in Older Patients with Dementia: A Systematic Review

as poor [25]. For further analysis of neuropsychological and physical exercise tests in this review, the study quality of at least 5 RCTs must be good or excellent.

\section{Reliability and Validity of Frequently Used Tests}

After identifying the tests that were used in $\geq 5$ good or excellent quality RCTs, a second search in PubMed was conducted through September 2011 to select the studies aimed at reporting the reliability and validity of these tests as evidenced in a population with dementia. Searches were performed by combining the terms 'reliability' OR 'validity' OR 'reproducibility of results' in combination with (with 'AND') keywords for dementia and the selected neuropsychological and physical exercise tests. By means of references, additional reliability and validity studies were searched.

\section{Results}

\section{Study Characteristics}

The literature searches for RCTs yielded a total of 840 potentially relevant publications. Eventually, 178 publications were full text screened of which 89 were excluded. A flowchart of the process is presented in figure 1 . The results are described in two separate sections: (1) neuropsychological tests and (2) exercise tests. These sections describe the test use in RCTs (number of RCTs), test use related to intervention type, test use related to dementia type, ESs measured with the tests, and study quality of RCTs (PEDro). Table 1 describes 59 neuropsychological tests that covered the cognitive domains global cognition, executive functioning, memory, and attention. Thereafter, table 2 describes the psychometric data of the neuropsychological tests that were most often used. Finally, 10 exercise tests that covered the physical domains endurance capacity, muscle strength, balance, and mobility are presented in table 3 .

\section{Neuropsychological Tests}

Frequency of Test Use

As is shown in table 1, global cognitive functioning was measured most often with the Mini Mental State Examination (MMSE) $(n=54)$, Alzheimer's Disease Assessment Scale cognitive subscale (ADAS-cog) $(n=43)$, and the Severe Impairment Battery (SIB) $(n=8)$. Tests for global cognitive functioning were used more often in comparison with neuropsychological tests that covered a specific cognitive area.

Thirty-two domain-specific neuropsychological tests were used in 63 RCTs, of which 7 tests were used in $\geq 5$ RCTs. Executive functioning was measured with the Verbal Fluency Test Category $(n=12)$, Clock Drawing Test $(n=6)$, Verbal Fluency Test Letters $(n=6)$, and the Trail Making Test-B $(n=5)$. Attention was measured with the Digit Span Forward $(n=$ 8), Digit Span Backward ( $\mathrm{n}=7)$, and Trail Making Test-A $(\mathrm{n}=6)$.

In summary, global cognitive tests were used more often than neuropsychological tests that covered a specific cognitive area. Frequently used neuropsychological tests that were used in $\geq 5$ RCTs covered the cognitive domains executive functioning and attention. Tests that were used in $>5$ RCTs which measured the cognitive domain memory were not found.

Dementia Type

A majority of the participants were diagnosed with Alzheimer's disease (AD; 84\%) or vascular dementia (VaD; 7\%) (table 1). Neuropsychological tests that were only administered in RCTs with AD patients were the SIB (global cognitive functioning), Verbal Fluency Test Letters (executive functioning), Trail Making Test-A, Digit Span Forward, and Digit Span Backward (attention). Tests used in RCTs with AD or VaD patients were the MMSE and 
Bossers et al.: Recommended Measures for the Assessment of Cognitive and Physical

Performance in Older Patients with Dementia: A Systematic Review

Fig. 1. Flow chart of literature

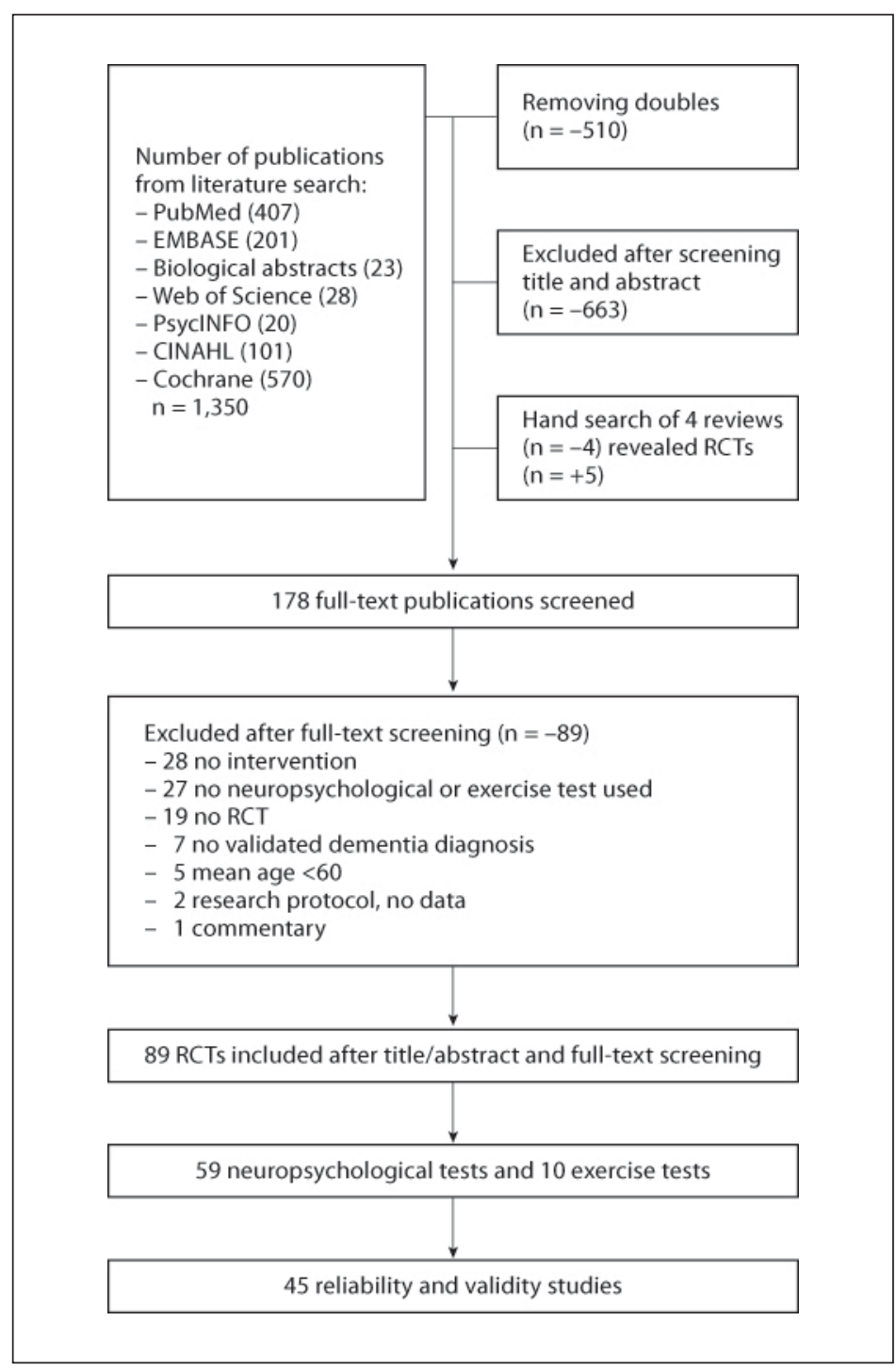
search and study selection.

ADAS-cog (global cognitive functioning), Verbal Fluency Test Category, Clock Drawing Test, and Trail Making Test-B (executive functioning). Tests that were used in RCTs with only $\mathrm{VaD}$, Lewy body disease, Pick's disease, and frontotemporal dementia patients were not found.

\section{Effect Size}

Pooled ESs ranged from small $(d=-0.16)$ to large $(d=1.58)$. The global cognitive test Rapid Evaluation of Cognitive Functioning measured a large ES $(d=1.12)$. The global cognitive tests SIB $(d=0.34)$, ADAS- $\operatorname{cog}(d=0.19)$, and MMSE $(d=0.09)$ showed small overall ESs. Overall ESs were small for both pharmacological and non-pharmacological RCTs. Furthermore, two neuropsychological tests that measured memory revealed large pooled ESs with the Visual Reproduction Test $(d=1.58)$ and the Syndrome Kurtz Test $(d=0.82)$. The Verbal Fluency Test Category that measures executive functioning measured a medium pooled ES $(d=0.61)$. 
Performance in Older Patients with Dementia: A Systematic Review

Table 1. Frequency of use of 59 neuropsychological tests (cognitive domain), descriptive statistics of the populations and RCTs in which these tests were used, the overall Cohen's $d$ ES (small/medium/large) for the tests in these RCTs, and range of the study quality (PEDro) of RCTs ( $\mathrm{n}=63$ in total) that used a given test

\begin{tabular}{|c|c|c|c|c|c|c|c|c|c|}
\hline $\begin{array}{l}\text { Neuropsychological test } \\
\text { (test domain) }\end{array}$ & $\begin{array}{l}\text { Stud- } \\
\text { ies, } \mathrm{n}\end{array}$ & $\begin{array}{l}\text { Partici- } \\
\text { pants, } n\end{array}$ & $\begin{array}{l}\text { Intervention type } \\
\text { (n of RCTs) }\end{array}$ & $\begin{array}{l}\text { Mean } \\
\text { age }^{\mathrm{a}} \pm \mathrm{SD} \\
\text { years }\end{array}$ & $\begin{array}{l}\text { Gen- } \\
\text { der } \\
\% \text { o }\end{array}$ & $\begin{array}{l}\text { Dementia } \\
\text { type }(\%)\end{array}$ & $\begin{array}{l}\text { Mean } \\
\text { baseline } \\
\pm \mathrm{SD}\end{array}$ & $\mathrm{ES}^{\mathrm{a}}$ & PEDro \\
\hline $\begin{array}{l}\text { MMSE (global functioning) } \\
{[26-79]}\end{array}$ & 54 & 7,606 & $\begin{array}{l}\text { pharmacological (39), } \\
\text { cognitive (9), exercise (5), } \\
\text { acupuncture }(1)\end{array}$ & $75.8 \pm 6.7$ & 58 & $\begin{array}{l}\operatorname{AD}(80) \\
\operatorname{VaD}(18) \\
\mathrm{LB}(2)\end{array}$ & $8.0 \pm 4.0$ & small & $\begin{array}{l}\text { E (6), G } \\
(45), \text { Mo } \\
(3), P(0)\end{array}$ \\
\hline $\begin{array}{l}\text { ADAS-cog (global functioning) } \\
{[26-28,31,32,35,39,41,43,} \\
47,48,51,54,55,58,61,63,64 \\
67,68,70-72,75,77,80-97]\end{array}$ & 43 & 10,133 & $\begin{array}{l}\text { pharmacological (37), } \\
\text { cognitive (5), exercise (1) }\end{array}$ & $74.4 \pm 6.7$ & 59 & $\begin{array}{l}\operatorname{AD}(81) \\
\operatorname{VaD}(17) \\
\mathrm{LB}(2)\end{array}$ & $24.4 \pm 11.0$ & small & $\begin{array}{l}\text { E (12), G } \\
(28), \text { Mo } \\
(3), P(0)\end{array}$ \\
\hline $\begin{array}{l}\text { Verbal Fluency Test Category } \\
(\mathrm{EF})[31,36,46,47,56,98-104]\end{array}$ & 12 & 726 & $\begin{array}{l}\text { pharmacological (5), } \\
\text { cognitive }(2), \text { exercise }(1) \text {, } \\
\text { hand move-ment }(1), \\
\text { airway stimuli ( } 1), \text { CES } \\
(1), \text { nerve stimuli }(1)\end{array}$ & $74.2 \pm 7.1$ & 56 & $\begin{array}{l}\operatorname{AD}(47) \\
\operatorname{VaD}(29) \\
\text { n.r. }(24)\end{array}$ & $10.9 \pm 9.2$ & medium & $\begin{array}{l}\text { E (4), G } \\
(6), M o \\
(1), P(1)\end{array}$ \\
\hline $\begin{array}{l}\text { SIB (global functioning) } \\
{[30,37,66,73,74,105-107]}\end{array}$ & 8 & 2,134 & pharmacological (8) & $76.6 \pm 7.7$ & 67 & $\mathrm{AD}(100)$ & $69.3 \pm 19.2$ & small & $\begin{array}{l}\text { E (3), G } \\
(5), \text { Mo } \\
(0), P(0)\end{array}$ \\
\hline $\begin{array}{l}\text { Digit Span Forward (attention) } \\
{[31,33,49,99,101-104]}\end{array}$ & 8 & 342 & $\begin{array}{l}\text { pharmacological ( } 1) \text {, } \\
\text { cognitive }(2), \text { exercise }(1) \text {, } \\
\text { hand move-ment (1), } \\
\text { airway stimuli (1), CES } \\
(1), \text { nerve stimuli (1) }\end{array}$ & $82.6 \pm 6.7$ & 55 & $\begin{array}{l}\mathrm{AD}(76) \\
\text { n.r. }(24)\end{array}$ & $5.4 \pm 2.3$ & small & $\begin{array}{l}\text { E (3), G } \\
(4), \text { Mo } \\
(0), P(1)\end{array}$ \\
\hline $\begin{array}{l}\text { Digit Span Backward (attention) } \\
{[31,49,99,101-104]}\end{array}$ & 7 & 280 & $\begin{array}{l}\text { cognitive (2), exercise (1), } \\
\text { hand movement (1), } \\
\text { airway stimuli (1), CES } \\
(1), \text { nerve stimuli (1) }\end{array}$ & $82.2 \pm 6.5$ & 50 & $\begin{array}{l}\mathrm{AD}(44) \\
\text { n.r. }(56)\end{array}$ & $3.9 \pm 1.5$ & small & $\begin{array}{l}\text { E (3), G } \\
(3), M o \\
(0), P(1)\end{array}$ \\
\hline $\begin{array}{l}\text { Clock Drawing Test (EF) } \\
{[42,50,56,95,100,108]}\end{array}$ & 6 & 1,674 & $\begin{array}{l}\text { pharmacological (5), } \\
\text { exercise (1) }\end{array}$ & $72.1 \pm 7.7$ & 68 & $\begin{array}{l}\operatorname{AD}(82) \\
\operatorname{VaD}(17) \\
\mathrm{LB}(1)\end{array}$ & $4.5 \pm 3.2$ & small & $\begin{array}{l}\mathrm{E}(3), \mathrm{G} \\
(2), \mathrm{M}(0), \\
\mathrm{P}(1)\end{array}$ \\
\hline $\begin{array}{l}\text { Trail Making Test-A } \\
\text { (attention) } \\
{[31,42,83,101-103]} \\
\end{array}$ & 6 & 1,502 & $\begin{array}{l}\text { pharmacological (3), } \\
\text { cognitive (1), airway } \\
\text { stimuli (1), CES (1) }\end{array}$ & $73.8 \pm 8.0$ & 67 & $\begin{array}{l}\text { AD (98), } \\
\text { FTD (2) }\end{array}$ & $161 \pm 82.5$ & small & $\begin{array}{l}\text { E (1), G } \\
(4), \text { Mo } \\
(0), P(1)\end{array}$ \\
\hline $\begin{array}{l}\text { Verbal Fluency Test Letters } \\
(\mathrm{EF})[36,56,99,101-103]\end{array}$ & 6 & 319 & $\begin{array}{l}\text { pharmacological (1), } \\
\text { cognitive (1), exercise (1), } \\
\text { airway stimuli (1), CES } \\
(1), \text { nerve stimuli }(1)\end{array}$ & $78.9 \pm 5.6$ & 44 & $\begin{array}{l}\mathrm{AD}(56) \\
\mathrm{PD}(44)\end{array}$ & $16.3 \pm 4.6$ & small & $\begin{array}{l}\text { E (2), G } \\
(3), \text { Mo } \\
(0), P(1)\end{array}$ \\
\hline $\begin{array}{l}\text { Trail Making Test-B (EF) } \\
{[27,31,47,98,101]}\end{array}$ & 5 & 214 & $\begin{array}{l}\text { pharmacological (3), } \\
\text { cognitive (1), airway } \\
\text { stimuli (1) }\end{array}$ & $73.9 \pm 6.2$ & 58 & $\mathrm{AD}(100)$ & $242.8 \pm 91.3$ & small & $\begin{array}{l}\text { E (2), G } \\
(3), \text { Mo } \\
(0), P(0)\end{array}$ \\
\hline $\begin{array}{l}\text { Logical Memory Test - } \\
\text { immediate recall (memory) } \\
{[28,49,69,70]}\end{array}$ & 4 & 277 & $\begin{array}{l}\text { pharmacological (2), } \\
\text { cognitive }(2)\end{array}$ & $75.6 \pm 6.8$ & 69 & $\mathrm{AD}(100)$ & $11.5 \pm 4.7$ & small & $\begin{array}{l}\text { E (2), G } \\
(2), \text { Mo } \\
(0), P(0)\end{array}$ \\
\hline $\begin{array}{l}\text { Logical Memory Test - } \\
\text { delayed recognition (memory) } \\
{[28,49,69,70]}\end{array}$ & 4 & 277 & $\begin{array}{l}\text { pharmacological (2), } \\
\text { cognitive }(2)\end{array}$ & $75.6 \pm 6.8$ & 69 & $\mathrm{AD}(100)$ & $2.8 \pm 2.9$ & small & $\begin{array}{l}\text { E (2), G } \\
(2), \text { Mo } \\
(0), P(0)\end{array}$ \\
\hline $\begin{array}{l}\text { Logical Memory Test - } \\
\text { delayed recall (memory) } \\
{[28,49,69,70]}\end{array}$ & 4 & 277 & $\begin{array}{l}\text { pharmacological (2), } \\
\text { cognitive (2) }\end{array}$ & $75.6 \pm 6.8$ & 69 & $\mathrm{AD}(100)$ & $0.9 \pm 1.6$ & small & $\begin{array}{l}\text { E (2), G } \\
(2), \text { Mo } \\
(0), P(0)\end{array}$ \\
\hline $\begin{array}{l}\text { Eight Word Test - immediate } \\
\text { recall (memory) }[99,102-104]\end{array}$ & 4 & 196 & $\begin{array}{l}\text { exercise (1), hand } \\
\text { movement (1), CES (1), } \\
\text { nerve stimuli (1) }\end{array}$ & $84.7 \pm 6.1$ & 62 & $\begin{array}{l}\mathrm{AD}(38) \\
\text { n.r. }(62)\end{array}$ & $17.6 \pm 8.1$ & small & $\begin{array}{l}\text { E (2), G } \\
(1), \text { Mo } \\
(0), P(1)\end{array}$ \\
\hline $\begin{array}{l}\text { Eight Word Test - delayed } \\
\text { recall (memory) }[99,102-104]\end{array}$ & 4 & 196 & $\begin{array}{l}\text { exercise (1), hand } \\
\text { movement (1), CES (1), } \\
\text { nerve stimuli (1) }\end{array}$ & $84.7 \pm 6.1$ & 62 & $\begin{array}{l}\mathrm{AD}(38) \\
\text { n.r. }(62)\end{array}$ & $0.35 \pm 0.94$ & small & $\begin{array}{l}\text { E (2), G } \\
(1), \text { Mo } \\
(0), P(1)\end{array}$ \\
\hline $\begin{array}{l}\text { Eight Word Test - delayed } \\
\text { recognition (memory) } \\
{[99,102-104]}\end{array}$ & 4 & 196 & $\begin{array}{l}\text { exercise (1), hand } \\
\text { movement (1), CES (1), } \\
\text { nerve stimuli (1) }\end{array}$ & $84.7 \pm 6.1$ & 62 & $\begin{array}{l}\mathrm{AD}(38) \\
\text { n.r. }(62)\end{array}$ & $10.7 \pm 3.9$ & small & $\begin{array}{l}\text { E (2), G } \\
(1), \text { Mo } \\
(0), P(1)\end{array}$ \\
\hline $\begin{array}{l}\text { Rivermead Behavioral Memory } \\
\text { Test - face recognition } \\
\text { (memory) }[99,102-104]\end{array}$ & 4 & 196 & $\begin{array}{l}\text { exercise (1), hand } \\
\text { movement (1), CES (1), } \\
\text { nerve stimuli (1) }\end{array}$ & $84.6 \pm 6.1$ & 59 & $\begin{array}{l}\mathrm{AD}(54) \\
\text { n.r. }(46)\end{array}$ & $6.6 \pm 3.5$ & small & $\begin{array}{l}\text { E (2), G } \\
(1), \text { Mo } \\
(0), P(1)\end{array}$ \\
\hline $\begin{array}{l}\text { Rivermead Behavioral Memory } \\
\text { Test - picture recognition } \\
\text { (memory) }[99,102-104]\end{array}$ & 4 & 196 & $\begin{array}{l}\text { exercise (1), hand } \\
\text { movement (1), CES (1), } \\
\text { nerve stimuli (1) }\end{array}$ & $84.6 \pm 6.1$ & 59 & $\begin{array}{l}\mathrm{AD}(54) \\
\text { n.r. }(46)\end{array}$ & $12.2 \pm 6.3$ & small & $\begin{array}{l}\text { E (2), G } \\
(1), \text { Mo } \\
(0), P(1)\end{array}$ \\
\hline
\end{tabular}




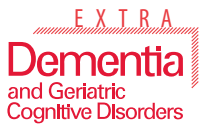

Dement Geriatr Cogn Disord Extra 2012;2:589-609

DOI: 10.1159/000345038

Published online: December 8, 2012

Bossers et al.: Recommended Measures for the Assessment of Cognitive and Physica

(c) 2012 S. Karger AG, Base

www.karger.com/dee

Table 1 (continued)

\begin{tabular}{|c|c|c|c|c|c|c|c|c|c|}
\hline $\begin{array}{l}\text { Neuropsychological test } \\
\text { (test domain) }\end{array}$ & $\begin{array}{l}\text { Stud- } \\
\text { ies, } n\end{array}$ & $\begin{array}{l}\text { Partici- } \\
\text { pants, } \mathrm{n}\end{array}$ & $\begin{array}{l}\text { Intervention type } \\
\text { (n of RCTs) }\end{array}$ & $\begin{array}{l}\text { Mean } \\
\text { age }^{a} \pm \mathrm{SD} \\
\text { years }\end{array}$ & $\begin{array}{l}\text { Gen- } \\
\text { der } \\
\% \text { ㅇ }\end{array}$ & $\begin{array}{l}\text { Dementia } \\
\text { type }(\%)\end{array}$ & $\begin{array}{l}\text { Mean } \\
\text { baseline }^{\mathrm{a}} \\
\pm \mathrm{SD}\end{array}$ & $\mathrm{ES}^{\mathrm{a}}$ & PEDro \\
\hline $\begin{array}{l}\text { Mattis Dementia Rating Scale } \\
\text { (global functioning) }[46,50, \\
101]\end{array}$ & 3 & 105 & $\begin{array}{l}\text { pharmacological (2), } \\
\text { airway stimuli (1) }\end{array}$ & $77.1 \pm 7.3$ & 67 & $\begin{array}{l}\mathrm{AD}(78) \\
\mathrm{LB}(22)\end{array}$ & $108.8 \pm 15.7$ & small & $\begin{array}{l}\mathrm{E}(1), \mathrm{G} \\
(2), \mathrm{Mo} \\
(0), \mathrm{P}(0)\end{array}$ \\
\hline $\begin{array}{l}\text { Modified Boston Naming Test } \\
\text { (language) }[31,49,69]\end{array}$ & 3 & 62 & $\begin{array}{l}\text { pharmacological (1), } \\
\text { cognitive }(2)\end{array}$ & $77.4 \pm 7.8$ & 76 & $\mathrm{AD}(100)$ & $10.4 \pm 4.6$ & small & $\begin{array}{l}\text { E (1), G } \\
(2), \text { Mo } \\
(0), P(0)\end{array}$ \\
\hline $\begin{array}{l}\text { Syndrome Kurtz Test } \\
\text { (attention/memory) }[52,100]\end{array}$ & 2 & 290 & pharmacological (2) & $65.2 \pm 7.5$ & 64 & $\begin{array}{l}\mathrm{AD}(38) \\
\operatorname{VaD}(62)\end{array}$ & $16.0 \pm 3.7$ & large & $\begin{array}{l}\text { E (2), G } \\
(0), \text { Mo } \\
(0), P(0)\end{array}$ \\
\hline $\begin{array}{l}\text { Digit Symbol Test (attention) } \\
{[42,101]}\end{array}$ & 2 & 273 & $\begin{array}{l}\text { pharmacological (1), } \\
\text { airway stimuli (1) }\end{array}$ & $73.8 \pm 8.0$ & 72 & $\mathrm{AD}(100)$ & $11.7 \pm 5.8$ & small & $\begin{array}{l}\text { E (1), G } \\
(1), \text { Mo } \\
(0), P(0)\end{array}$ \\
\hline $\begin{array}{l}\text { STROOP color-word } \\
\text { interference (EF) }[98,101]\end{array}$ & 2 & 67 & $\begin{array}{l}\text { pharmacological (1), } \\
\text { airway stimuli (1) }\end{array}$ & $77.7 \pm 6.6$ & 54 & $\mathrm{AD}(100)$ & $65.5 \pm 41.2$ & small & $\begin{array}{l}\text { E (1), G } \\
(1), \text { Mo } \\
(0), P(0)\end{array}$ \\
\hline $\begin{array}{l}\text { Cambridge Neuropsychological } \\
\text { Test Battery (global } \\
\text { functioning) }[78,109]\end{array}$ & 2 & 50 & $\begin{array}{l}\text { pharmacological (1), } \\
\text { exercise stimuli (1) }\end{array}$ & $71.2 \pm 8.1$ & 56 & $\begin{array}{l}\text { AD (65), } \\
\text { FTD (35) }\end{array}$ & - & - & $\begin{array}{l}\mathrm{E}(0), \mathrm{G} \\
(1), \mathrm{Mo} \\
(1), \mathrm{P}(0)\end{array}$ \\
\hline $\begin{array}{l}\text { Visual Memory Span } \\
\text { (memory) }[102,103]\end{array}$ & 2 & 38 & CES (1), nerve stimuli (1) & $84.4 \pm 6.3$ & 59 & $\mathrm{AD}(100)$ & $9.0 \pm 3.8$ & small & $\begin{array}{l}\text { E (0), G } \\
(1), \text { Mo } \\
(0), P(1)\end{array}$ \\
\hline $\begin{array}{l}\text { Selective Reminding Test } \\
\text { (memory) }[28,49]\end{array}$ & 2 & 35 & cognitive (2) & $72.9 \pm 7.1$ & 89 & $\mathrm{AD}(100)$ & $13.3 \pm 12.4$ & small & $\begin{array}{l}\text { E (2), G } \\
(0), \text { Mo } \\
(0), P(0)\end{array}$ \\
\hline Block Design Test (EF) $[84,98]$ & 2 & 31 & pharmacological (2) & $72.8 \pm 6.8$ & 0 & $\mathrm{AD}(100)$ & $45.5 \pm 14.9$ & small & $\begin{array}{l}\text { E (2), G } \\
(0), \text { Mo } \\
(0), P(0)\end{array}$ \\
\hline $\begin{array}{l}\text { The Executive Interview (EF) } \\
\text { [81] }\end{array}$ & 1 & 363 & pharmacological (1) & $72.3 \pm 9.0$ & 38 & $\mathrm{VaD}(100)$ & $18.3 \pm 7.0$ & small & $\begin{array}{l}\mathrm{E}(0), \mathrm{G} \\
(1), \mathrm{Mo} \\
(0), \mathrm{P}(0)\end{array}$ \\
\hline $\begin{array}{l}\text { Cambridge Cognitive } \\
\text { Examination (global } \\
\text { functioning) [38] }\end{array}$ & 1 & 179 & pharmacological (1) & $87.4 \pm 6.0$ & 57 & $\mathrm{AD}(100)$ & $69.0 \pm 13.0$ & small & $\begin{array}{l}\text { E (0), G } \\
(1), \text { Mo } \\
(0), P(0)\end{array}$ \\
\hline $\begin{array}{l}\text { Age-Adjusted Concentration } \\
\text { Task (attention) [65] }\end{array}$ & 1 & 65 & pharmacological (1) & $77.8 \pm 5.6$ & 58 & $\begin{array}{l}\mathrm{AD}(66) \\
\operatorname{VaD}(11)\end{array}$ & - & small & $\begin{array}{l}\text { E (0), G } \\
(1), \text { Mo } \\
(0), P(0)\end{array}$ \\
\hline $\begin{array}{l}\text { Auditory Verbal Learning } \\
\text { Test - Chinese version } \\
\text { (memory) [33] }\end{array}$ & 1 & 62 & pharmacological (1) & $83.9 \pm 7.6$ & 80 & $\mathrm{AD}(100)$ & $2.6 \pm 1.5$ & small & $\begin{array}{l}\text { E (0), G } \\
(0), \text { Mo } \\
(1), P(0)\end{array}$ \\
\hline $\begin{array}{l}\text { Stop Signal Reaction Time } \\
\text { (attention) [104] }\end{array}$ & 1 & 61 & hand movement (1) & $84.7 \pm 5.1$ & 58 & n.r. & - & - & $\begin{array}{l}\text { E (0), G } \\
(0), \text { Mo } \\
(1), P(0)\end{array}$ \\
\hline $\begin{array}{l}\text { Attention Network Task } \\
\text { (attention) [104] }\end{array}$ & 1 & 61 & hand movement (1) & $84.7 \pm 5.1$ & 58 & n.r. & - & - & $\begin{array}{l}\text { E (0), G } \\
(0), \text { Mo } \\
(1), P(0)\end{array}$ \\
\hline $\begin{array}{l}\text { Hasegawa's Evaluation of } \\
\text { Cognitive Functioning (global } \\
\text { functioning) [76] }\end{array}$ & 1 & 60 & acupuncture (1) & $66.7 \pm 10.5$ & 34 & $\mathrm{VaD}(100)$ & $11.3 \pm 4.5$ & small & $\begin{array}{l}\text { E (0), G } \\
(0), \text { Mo } \\
(1), P(0)\end{array}$ \\
\hline $\begin{array}{l}\text { Cognitive Abilities Screening } \\
\text { Instrument (global } \\
\text { functioning) [40] }\end{array}$ & 1 & 60 & cognitive (1) & $82.3 \pm 5.9$ & 67 & $\mathrm{VaD}(100)$ & $54.6 \pm 15.3$ & small & $\begin{array}{l}\text { E (1), G } \\
(0), \text { Mo } \\
(0), P(0)\end{array}$ \\
\hline $\begin{array}{l}\text { Digit Cancellation Task } \\
\text { (attention) [101] }\end{array}$ & 1 & 52 & airway stimuli (1) & $78.2 \pm 7.2$ & 69 & $\mathrm{AD}(100)$ & - & - & $\begin{array}{l}\text { E (0), G } \\
(0), \text { Mo } \\
(1), P(0)\end{array}$ \\
\hline $\begin{array}{l}\text { Hopkins Verbal Learning } \\
\text { Test - revised (memory) } \\
{[101]}\end{array}$ & 1 & 52 & airway stimuli (1) & $78.2 \pm 7.2$ & 69 & $\mathrm{AD}(100)$ & $3.3 \pm 1.5$ & small & $\begin{array}{l}\text { E (0), G } \\
(1), \text { Mo } \\
(0), P(0)\end{array}$ \\
\hline $\begin{array}{l}\text { Wisconsin Card Sorting Test } \\
\text { (EF) }[101]\end{array}$ & 1 & 52 & airway stimuli (1) & $78.2 \pm 7.2$ & 69 & $\mathrm{AD}(100)$ & - & - & $\begin{array}{l}\text { E (0), G } \\
(1), \text { Mo } \\
(0), P(0)\end{array}$ \\
\hline $\begin{array}{l}\text { The Executive Clock Drawing } \\
\text { Task } 1 \text { (EF) [110] }\end{array}$ & 1 & 51 & pharmacological (1) & $77.9 \pm 7.0$ & 55 & $\mathrm{AD}(100)$ & $6.2 \pm 3.7$ & small & $\begin{array}{l}\text { E (0), G } \\
(1), \text { Mo } \\
(0), P(0)\end{array}$ \\
\hline $\begin{array}{l}\text { The Executive Clock Drawing } \\
\text { Task } 2 \text { (EF) [110] }\end{array}$ & 1 & 51 & pharmacological (1) & $77.9 \pm 7.0$ & 55 & $\mathrm{AD}(100)$ & $10.7 \pm 3.0$ & small & $\begin{array}{l}\text { E (0), G } \\
(1), \text { Mo } \\
(0), P(0)\end{array}$ \\
\hline
\end{tabular}




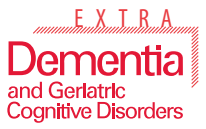

Dement Geriatr Cogn Disord Extra 2012;2:589-609

DOI: 10.1159/000345038

Published online: December 8, 2012

Bossers et al.: Recommended Measures for the Assessment of Cognitive and Physical

C 2012 S. Karger AG, Basel

www.karger.com/dee

Table 1 (continued)

\begin{tabular}{|c|c|c|c|c|c|c|c|c|c|}
\hline $\begin{array}{l}\text { Neuropsychological test } \\
\text { (test domain) }\end{array}$ & $\begin{array}{l}\text { Stud- } \\
\text { ies, n }\end{array}$ & $\begin{array}{l}\text { Partici- } \\
\text { pants, } n\end{array}$ & $\begin{array}{l}\text { Intervention type } \\
\text { (n of RCTs) }\end{array}$ & $\begin{array}{l}\text { Mean } \\
\text { age }^{a} \pm S D \\
\text { years }\end{array}$ & $\begin{array}{l}\text { Gen- } \\
\text { der } \\
\% q\end{array}$ & $\begin{array}{l}\text { Dementia } \\
\text { type }(\%)\end{array}$ & $\begin{array}{l}\text { Mean } \\
\text { baseline }^{\mathrm{a}} \\
\pm \mathrm{SD}\end{array}$ & $\mathrm{ES}^{\mathrm{a}}$ & PEDro \\
\hline $\begin{array}{l}\text { Rey-Osterrieth Complex } \\
\text { Figure Test - Copy (EF) [36] }\end{array}$ & 1 & 32 & cognitive (1) & $73.0 \pm 7.2$ & 62 & $\mathrm{AD}(100)$ & $16.5 \pm 14.6$ & small & $\begin{array}{l}\mathrm{E}(0), \mathrm{G} \\
(1), \text { Mo } \\
(0), \mathrm{P}(0)\end{array}$ \\
\hline $\begin{array}{l}\text { Rey-Osterrieth Complex } \\
\text { Figure Test - Recall (EF) [36] }\end{array}$ & 1 & 32 & cognitive (1) & $73.0 \pm 7.2$ & 62 & $\mathrm{AD}(100)$ & $1.2 \pm 2.0$ & small & $\begin{array}{l}\text { E (0), G } \\
(1), \text { Mo } \\
(0), P(0)\end{array}$ \\
\hline $\begin{array}{l}\text { Three Dimensional } \\
\text { Constructional Praxis } \\
\text { (constructive abilities) [69] }\end{array}$ & 1 & 32 & cognitive (1) & $73.0 \pm 7.2$ & 62 & $\mathrm{AD}(100)$ & $11.9 \pm 0.48$ & - & $\begin{array}{l}\text { E (0), G } \\
(1), \text { Mo } \\
(0), P(0)\end{array}$ \\
\hline $\begin{array}{l}\text { Extended Rivermead } \\
\text { Behavioral Memory Test - } \\
\text { profile (memory) [36] }\end{array}$ & 1 & 32 & cognitive (1) & $73.0 \pm 7.2$ & 62 & $\mathrm{AD}(100)$ & $1.2 \pm 1.3$ & small & $\begin{array}{l}\text { E (0), G } \\
(1), \text { Mo } \\
(0), P(0)\end{array}$ \\
\hline $\begin{array}{l}\text { Attention Matrices Test } \\
\text { (attention) [36] }\end{array}$ & 1 & 32 & cognitive (1) & $73.0 \pm 7.2$ & 62 & $\mathrm{AD}(100)$ & $32.4 \pm 11.7$ & small & $\begin{array}{l}\mathrm{E}(0), \mathrm{G} \\
(1), \text { Mo } \\
(0), \mathrm{P}(0)\end{array}$ \\
\hline $\begin{array}{l}\text { Visual Reproduction } 1 \\
\text { (memory) [28] }\end{array}$ & 1 & 16 & cognitive (1) & $73.8 \pm 4.8$ & 82 & $\mathrm{AD}(100)$ & $13.8 \pm 3.6$ & large & $\begin{array}{l}\text { E (1), G } \\
(0), \text { Mo } \\
(0), P(0)\end{array}$ \\
\hline $\begin{array}{l}\text { Rapid Evaluation of Cognitive } \\
\text { Functioning (global } \\
\text { functioning) [111] }\end{array}$ & 1 & 31 & exercise (1) & $81.8 \pm 5.3$ & 74 & $\mathrm{AD}(100)$ & $27.6 \pm 6.8$ & large & $\begin{array}{l}\text { E (0), G } \\
(1), \text { Mo } \\
(0), P(0)\end{array}$ \\
\hline $\begin{array}{l}\text { Developmental Test of Visual } \\
\text { Motor Integration (perception) } \\
{[84]}\end{array}$ & 1 & 16 & pharmacological (1) & $69.8 \pm 8.6$ & 0 & $\mathrm{AD}(100)$ & $18.1 \pm 2.7$ & small & $\begin{array}{l}\text { E (1), G } \\
(0), \text { Mo } \\
(0), P(0)\end{array}$ \\
\hline $\begin{array}{l}\text { Visual Reproduction } 2 \\
\text { (memory) [28] }\end{array}$ & 1 & 16 & cognitive (1) & $73.8 \pm 4.8$ & 82 & $\mathrm{AD}(100)$ & $1.3 \pm 2.8$ & small & $\begin{array}{l}\text { E (1), G } \\
(0), \text { Mo } \\
(0), P(0)\end{array}$ \\
\hline $\begin{array}{l}\text { Judgment of Line Orientation } \\
\text { (constructive abilities) [84] }\end{array}$ & 1 & 16 & pharmacological (1) & $69.8 \pm 8.6$ & 0 & $\mathrm{AD}(100)$ & $18.2 \pm 8.9$ & small & $\begin{array}{l}\text { E (1), G } \\
(0), \text { Mo } \\
(0), P(0)\end{array}$ \\
\hline $\begin{array}{l}\text { California Verbal Learning } \\
\text { Test - delayed recall } \\
\text { (memory) [84] }\end{array}$ & 1 & 16 & pharmacological (1) & $69.8 \pm 8.6$ & 0 & $\mathrm{AD}(100)$ & $1.6 \pm 1.9$ & small & $\begin{array}{l}\text { E (1), G } \\
(0), \text { Mo } \\
(0), P(0)\end{array}$ \\
\hline $\begin{array}{l}\text { Recognition Memory Test - } \\
\text { faces (memory) [28] }\end{array}$ & 1 & 16 & cognitive (1) & $73.8 \pm 4.8$ & 82 & $\mathrm{AD}(100)$ & $28.0 \pm 5.9$ & small & $\begin{array}{l}\text { E (1), G } \\
(0), \text { Mo } \\
(0), P(0)\end{array}$ \\
\hline $\begin{array}{l}\text { Benton Visual Retention Test } \\
\text { (memory) [49] }\end{array}$ & 1 & 19 & cognitive (1) & $72.1 \pm 8.5$ & 95 & $\mathrm{AD}(100)$ & $1.9 \pm 1.8$ & small & $\begin{array}{l}\text { E (1), G } \\
(0), \text { Mo } \\
(0), P(0)\end{array}$ \\
\hline $\begin{array}{l}\text { Recognition Memory Test - } \\
\text { words (memory) [28] }\end{array}$ & 1 & 16 & cognitive (1) & $73.8 \pm 4.8$ & 82 & $\mathrm{AD}(100)$ & $32.7 \pm 8.9$ & small & $\begin{array}{l}\text { E (1), G } \\
(0), \text { Mo } \\
(0), P(0)\end{array}$ \\
\hline $\begin{array}{l}\text { Milan Overall Dementia } \\
\text { Assessment (global } \\
\text { functioning) [59] }\end{array}$ & 1 & 16 & cognitive (1) & $68.0 \pm 6.5$ & 48 & $\mathrm{AD}(100)$ & - & small & $\begin{array}{l}\text { E (0), G } \\
(0), \text { Mo } \\
(1), P(0)\end{array}$ \\
\hline $\begin{array}{l}\text { Proactive Interference Test } \\
\text { (memory) [98] }\end{array}$ & 1 & 15 & pharmacological (1) & $76.0 \pm 4.0$ & 0 & $\mathrm{AD}(100)$ & $7.7 \pm 4.2$ & small & $\begin{array}{l}\text { E (1), G } \\
(0), \text { Mo } \\
(0), P(0)\end{array}$ \\
\hline Route Test (EF) [98] & 1 & 15 & pharmacological (1) & $76.0 \pm 4.0$ & 0 & $\mathrm{AD}(100)$ & $15.1 \pm 9.6$ & small & $\begin{array}{l}\text { E (1), G } \\
(0), \text { Mo } \\
(0), P(0)\end{array}$ \\
\hline $\begin{array}{l}\text { Story Recall Test (memory) } \\
{[98]}\end{array}$ & 1 & 15 & pharmacological (1) & $76.0 \pm 4.0$ & 0 & $\mathrm{AD}(100)$ & $12.0 \pm 13.2$ & small & $\begin{array}{l}\text { E (1), G } \\
(0), \text { Mo } \\
(0), P(0)\end{array}$ \\
\hline $\begin{array}{l}\text { Fuld Object and Memory } \\
\text { Evaluation (memory) [31] }\end{array}$ & 1 & 13 & cognitive (1) & $73.3 \pm 6.4$ & 69 & $\mathrm{AD}(100)$ & $24.7 \pm 11.1$ & small & $\begin{array}{l}\text { E (0), G } \\
(1), \text { Mo } \\
(0), P(0)\end{array}$ \\
\hline
\end{tabular}

$\mathrm{E}=$ Excellent (9-10); G = good (6-8); Mo = moderate (4-5); $\mathrm{P}=$ poor (0-3); EF = executive functioning; CES = cranial electrostimulation; $\mathrm{LB}=$ Lewy body disease $\mathrm{PD}=$ Pick's disease FTD = frontotemporal dementia; n.r. = not reported. ${ }^{\text {a }}$ Pooled and weighted data as a function of the number of participants. 
Bossers et al.: Recommended Measures for the Assessment of Cognitive and Physica

Performance in Older Patients with Dementia: A Systematic Review

Table 2. Reliability, validity, and summary of the psychometric properties of 10 selected neuropsychological tests (cognitive domain) that were used in $\geq 5$ good- or high-quality RCTs

\begin{tabular}{|c|c|c|c|}
\hline $\begin{array}{l}\text { Neuropsychological } \\
\text { test (domain) }\end{array}$ & Reliability & Validity & Summary \\
\hline $\begin{array}{l}\text { MMSE } \\
\text { (global functioning) }\end{array}$ & $\begin{array}{l}\text { test-retest reliability (ICC): } 0.85-0.90[112] ; 0.89 \\
{[113] ; 0.92[114] ; 0.69[115] ; 0.89[116] ; 0.80[117] ;} \\
0.86[118] \\
\text { inter-rater reliability: ICC }=0.69-0.78[115] ; \\
\kappa=0.63[114] ; \text { ICC }=0.69[115] \\
\text { internal consistency }(\alpha): 0.54-0.96[119] ; 0.78[120] ; \\
0.77[121] ; 0.68[122] ; 0.96[123] ; 0.90[124] ; 0.81 \\
{[125]}\end{array}$ & $\begin{array}{l}\text { concurrent validity with } \\
\text { Wechsler adult intelligence } \\
\text { scale verbal IQ }(\mathrm{r}=0.78) \\
\text { and performance IQ } \\
(\mathrm{r}=0.66)[113]\end{array}$ & $\begin{array}{l}\text { reliable and valid test in dementia } \\
\text { patients; there is a floor effect in } \\
\text { severe dementia patients [119]; } \\
\text { sensitivity to change over time is } \\
\text { questionable because small changes } \\
\text { could be due to measurement errors } \\
\text { [125] }\end{array}$ \\
\hline $\begin{array}{l}\text { ADAS-cog } \\
\text { (global functioning) }\end{array}$ & 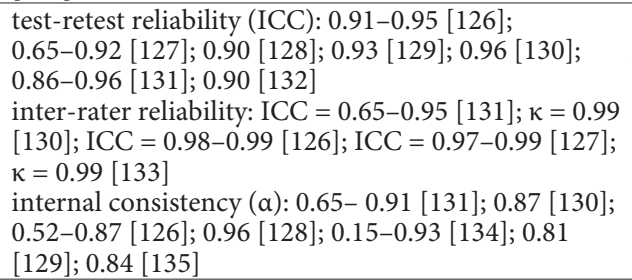 & $\begin{array}{l}\text { concurrent validity with } \\
\text { MMSE }(r=-0.63)[135]\end{array}$ & $\begin{array}{l}\text { reliable and valid test in patients } \\
\text { with mild to moderate dementia }\end{array}$ \\
\hline $\begin{array}{l}\text { Verbal Fluency Test } \\
\text { Category (EF) }\end{array}$ & - & - & $\begin{array}{l}\text { no information available about } \\
\text { reliability and validity for dementia } \\
\text { patients }\end{array}$ \\
\hline SIB (global functioning) & $\begin{array}{l}\text { test-retest reliability (ICC): } 0.79 \text { [136]; } 0.97 \text { [137]; } \\
0.87[138] ; 0.90 \text { [139]; } 0.93 \text { [135] } \\
\text { inter-rater reliability: Spearman's } \rho=0.85[140] \text {; } \\
\text { Spearman's } \rho=0.97 \text { [137]; ICC = 0.99 [138] } \\
\text { internal consistency }(\alpha): 0.97 \text { [136]; } 0.97 \text { [140]; } 0.98 \\
\text { [137] }\end{array}$ & $\begin{array}{l}\text { concurrent validity with } \\
\text { MMSE }(r=0.85)[140]\end{array}$ & $\begin{array}{l}\text { reliable and valid test in dementia } \\
\text { patients [140]; this test is sensitive to } \\
\text { changes in patients with moderate to } \\
\text { severe dementia (MMSE 0-12) } \\
\text { [140]; promising test for follow-up } \\
\text { in therapeutic trials [138] }\end{array}$ \\
\hline $\begin{array}{l}\text { Digit Span Forward } \\
\text { (attention) }\end{array}$ & - & - & $\begin{array}{l}\text { no information available about } \\
\text { reliability and validity for dementia } \\
\text { patients; Digit Span Test as a sub- } \\
\text { test in the SIB was sensitive to } \\
\text { change in dementia patients [141] }\end{array}$ \\
\hline $\begin{array}{l}\text { Verbal Fluency Test } \\
\text { Letters (EF) }\end{array}$ & - & - & $\begin{array}{l}\text { no information available about } \\
\text { reliability and validity for dementia } \\
\text { patients }\end{array}$ \\
\hline $\begin{array}{l}\text { Digit Span Backward } \\
\text { (attention) }\end{array}$ & - & - & $\begin{array}{l}\text { no information available about } \\
\text { reliability and validity for dementia } \\
\text { patients; Digit Span Test as a sub- } \\
\text { test in the SIB was sensitive to } \\
\text { change in dementia patients [141] }\end{array}$ \\
\hline $\begin{array}{l}\text { Clock Drawing Test } \\
\text { (EF) }\end{array}$ & $\begin{array}{l}\text { test-retest reliability (ICC): } 0.70-0.78[142] \\
\text { inter-rater reliability: ICC }=0.82[143] ; \text { ICC }=0.92 \\
{[144] ; \mathrm{ICC}=0.88[145] ; \kappa=0.82-0.94[146] ; \kappa=0.94} \\
{[147] ; \kappa=0.63-1.0[148]} \\
\text { internal consistency }(\alpha): 0.75[142]\end{array}$ & $\begin{array}{l}\text { concurrent validity with } \\
\text { MMSE }(r=0.13)[142]\end{array}$ & reliable test in dementia patients \\
\hline $\begin{array}{l}\text { Trail Making Test-A } \\
\text { (attention) }\end{array}$ & - & - & $\begin{array}{l}\text { no information available about } \\
\text { reliability and validity for dementia } \\
\text { patients }\end{array}$ \\
\hline $\begin{array}{l}\text { Trail Making Test-B } \\
\text { (EF) }\end{array}$ & - & - & $\begin{array}{l}\text { no information available about } \\
\text { reliability and validity for dementia } \\
\text { patients }\end{array}$ \\
\hline
\end{tabular}

$\mathrm{EF}=$ Executive functioning.

Study Quality

According to the PEDro scale, the study quality of RCTs that used neuropsychological tests ranged from 2 (poor) to 10 (excellent). Three RCTs with poor study quality used the Verbal Fluency Test Category, Digit Span Forward, Digit Span Backward, Clock Drawing Test, Trail Making Test-A, and Verbal Fluency Test Letters. Because these tests were also found in RCTs with excellent and good study quality, this had no effect on the selection process of these neuropsychological tests. 
Performance in Older Patients with Dementia: A Systematic Review

Table 3. Frequency of use of 10 physical exercise tests (physical exercise domain), descriptive statistics of the populations and RCTs in which these tests were used, the overall Cohen's d ES (small/medium/large) for the tests in these RCTs, and range of the study quality (PEDro) of RCTs $(n=13$ in total) that used a given test

\begin{tabular}{|c|c|c|c|c|c|c|c|c|c|}
\hline $\begin{array}{l}\text { Physical exercise test } \\
\text { (test domain) }\end{array}$ & $\begin{array}{l}\text { Stud- } \\
\text { ies, } n\end{array}$ & $\begin{array}{l}\text { Partici- } \\
\text { pants, } \mathrm{n}\end{array}$ & $\begin{array}{l}\text { Intervention } \\
\text { type } \\
\text { (n of RCTs) }\end{array}$ & $\begin{array}{l}\text { Mean age } \mathrm{a}^{\mathrm{a}} \\
\pm \mathrm{SD} \\
\text { years }\end{array}$ & $\begin{array}{l}\text { Gender } \\
\% \text { \% }\end{array}$ & Dementia type (\%) & $\begin{array}{l}\text { Mean } \\
\text { baseline }^{\mathrm{a}} \pm \\
\text { SD }\end{array}$ & $\mathrm{ES}^{\mathrm{a}}$ & PEDro \\
\hline $\begin{array}{l}\text { Timed Up and Go Test } \\
\text { (mobility) }[45,150,151]\end{array}$ & 3 & 179 & Exercise (3) & $81.9 \pm 7.3$ & 71 & $\mathrm{AD}(100)$ & $17.1 \pm 7.5$ & small & $\begin{array}{l}\mathrm{E}(0), \\
\mathrm{G}(2), \\
\text { Mo (1), } \\
\mathrm{P}(0)\end{array}$ \\
\hline $\begin{array}{l}\text { Six Minute Walk Distance } \\
\text { (endurance capacity) }[45,77]\end{array}$ & 2 & 105 & Exercise (2) & $77.6 \pm 6.6$ & 65 & $\begin{array}{l}\mathrm{AD}(39), \mathrm{VaD}(16) \\
\mathrm{LB}(16), \text { n.r. }(29)\end{array}$ & $221.0 \pm 82.6$ & medium & $\begin{array}{l}\mathrm{E}(0), \\
\mathrm{G}(2), \\
\text { Mo }(0), \\
\mathrm{P}(0)\end{array}$ \\
\hline $\begin{array}{l}\text { Functional Reach Test } \\
\text { (flexibility) }[77,150]\end{array}$ & 2 & 94 & Exercise (2) & $76.6 \pm 6.6$ & 52 & $\mathrm{AD}(82), \mathrm{VaD}(18)$ & $20.4 \pm 8.1$ & small & $\begin{array}{l}\mathrm{E}(0), \\
\mathrm{G}(2), \\
\mathrm{Mo}(0), \\
\mathrm{P}(0)\end{array}$ \\
\hline $\begin{array}{l}\text { Six Meter Walk (mobility) } \\
\text { [151] }\end{array}$ & 1 & 134 & Exercise (1) & $83.0 \pm 7.4$ & 75 & $\mathrm{AD}(100)$ & $0.4 \pm 0.2$ & medium & $\begin{array}{l}\text { E }(0), \\
G(1), \\
\text { Mo }(0), \\
P(0)\end{array}$ \\
\hline $\begin{array}{l}\text { Abnormal One-Leg Balance } \\
\text { (balance) [151] }\end{array}$ & 1 & 134 & Exercise (1) & $83.0 \pm 7.4$ & 75 & $\mathrm{AD}(100)$ & - & - & $\begin{array}{l}\text { E (0), } \\
\text { G }(1), \\
\text { Mo (0), } \\
P(0)\end{array}$ \\
\hline $\begin{array}{l}\text { Tinetti Balance Scale } \\
\text { (balance) [56] }\end{array}$ & 1 & 116 & $\begin{array}{l}\text { Pharma- } \\
\text { cological (1) }\end{array}$ & $73.4 \pm 2.5$ & 62 & $\mathrm{AD}(100)$ & $8.5 \pm 1.2$ & large & $\begin{array}{l}\mathrm{E}(1), \\
\mathrm{G}(0), \\
\text { Mo }(0), \\
\mathrm{P}(0)\end{array}$ \\
\hline $\begin{array}{l}\text { Five Times Sit to Stand } \\
\text { (leg strength) [150] }\end{array}$ & 1 & 29 & Exercise (1) & $76.9 \pm 6.7$ & 51 & $\mathrm{AD}(72), \mathrm{LB}(28)$ & $18.9 \pm 7.2$ & small & $\begin{array}{l}\mathrm{E}(0), \\
\mathrm{G}(1), \\
\text { Mo }(0), \\
\mathrm{P}(0)\end{array}$ \\
\hline $\begin{array}{l}\text { Berg Balance Scale (balance) } \\
{[77]}\end{array}$ & 1 & 85 & Exercise (1) & $76.6 \pm 6.5$ & 52 & $\begin{array}{l}\mathrm{AD}(61), \mathrm{VaD}(20) \\
\mathrm{LB}(19)\end{array}$ & $47.5 \pm 16.9$ & small & $\begin{array}{l}\mathrm{E}(0), \\
\mathrm{G}(1), \\
\text { Mo (0), } \\
\mathrm{P}(0)\end{array}$ \\
\hline $\begin{array}{l}30 \text { Second Chair Stand } \\
\text { (leg strength) [152] }\end{array}$ & 1 & 16 & Exercise (1) & $74.5 \pm-$ & 37 & $\mathrm{AD}(100)$ & - & - & $\begin{array}{l}\mathrm{E}(0), \\
\mathrm{G}(0), \\
\mathrm{Mo}(1), \\
\mathrm{P}(0)\end{array}$ \\
\hline $\begin{array}{l}\text { Two Minute Step Test } \\
\text { (endur-ance capacity) [152] }\end{array}$ & 1 & 16 & Exercise (1) & $74.5 \pm-$ & 37 & $\mathrm{AD}(100)$ & - & - & $\begin{array}{l}\mathrm{E}(0), \\
\mathrm{G}(0), \\
\mathrm{Mo}(1), \\
\mathrm{P}(0)\end{array}$ \\
\hline
\end{tabular}

$\mathrm{E}=$ Excellent (9-10); G = good (6-8); Mo = moderate (4-5); P = poor (0-3); LB = Lewy body disease; n.r. = not reported. ${ }^{\text {a }}$ Weighted data as a function of the number of participants.

Reliability and Validity

Table 2 presents the reliability and validity of 10 neuropsychological tests that were used in $\geq 5$ good or excellent RCTs. The global cognitive tests MMSE, ADAS-cog, and SIB were found to be reliable and valid tools for dementia patients. The Clock Drawing Test was reliable but showed an unsatisfactory concurrent validity with other tests that measured executive functioning [149]. No reliability or validity studies with dementia patients were found for the Verbal Fluency Test Category, Verbal Fluency Test Letters, Trail Making Test-B, Digit Span Forward, Digit Span Backward, and Trail Making Test-A. 
Table 4. Recommendations of global and specific neuropsychological tests ordered on the basis of frequency of test use, overall ES, study quality, reliability, and validity for global cognitive functioning, executive functioning, memory, and attention

\begin{tabular}{|c|c|c|c|c|}
\hline & Global functioning & Executive functioning & Memory & Attention \\
\hline 1 & $\operatorname{SIB}^{\mathrm{a}-\mathrm{d}}$ & $\begin{array}{l}\text { Verbal Fluency } \\
\text { Test Category }{ }^{\mathrm{a}-\mathrm{c}}\end{array}$ & $\begin{array}{l}\text { Visual Reproduction } \\
\text { Test }^{*, b, c}\end{array}$ & $\begin{array}{l}\text { Digit Span } \\
\text { Forward }^{\mathrm{a}, \mathrm{c}}\end{array}$ \\
\hline 2 & $\mathrm{MMSE}^{\mathrm{a}, \mathrm{c}, \mathrm{d}}$ & Clock Drawing Test ${ }^{\mathrm{a}, \mathrm{c}, \mathrm{d}}$ & Eight Word Test ${ }^{*, c}$ & $\begin{array}{l}\text { Digit Span } \\
\text { Backward }^{\mathrm{a}, \mathrm{c}}\end{array}$ \\
\hline 3 & ADAS- $\operatorname{cog}^{\mathrm{a}, \mathrm{c}, \mathrm{d}}$ & $\begin{array}{l}\text { Verbal Fluency Test } \\
\text { Letters } \mathrm{s}^{\mathrm{a}, \mathrm{c}}\end{array}$ & $\begin{array}{l}\text { Logical Memory } \\
\text { Test }^{*, c}\end{array}$ & $\begin{array}{l}\text { Trail Making } \\
\text { Test- } A^{\text {a, c }}\end{array}$ \\
\hline 4 & $\begin{array}{l}\text { Rapid Evaluation of } \\
\text { Cognitive Functioning*,b-d }\end{array}$ & Trail Making Test- $\mathrm{B}^{\mathrm{a}, \mathrm{c}}$ & & \\
\hline
\end{tabular}

${ }^{a}$ Frequently used in RCTs (feasibility). ${ }^{b}$ Able to measure an effect (sensitivity to change). ${ }^{c}$ Test was used in excellent/good quality RCTs (PEDro). ${ }^{\mathrm{d}}$ Reliable/valid in dementia patients.

* More research is needed to recommend these tests.

\section{Exercise Tests}

Frequency of Test Use

Ten different exercise tests were used in 13 RCTs (table 3). These tests measured the physical exercise domains endurance capacity with the Six Minute Walk Distance and Two Minute Step Test; muscle strength with the Five Times Sit To Stand and 30 Second Chair Stand; balance with the Tinetti Balance Scale, Abnormal One-Leg Balance, and Berg Balance Scale; mobility with the Timed Up and Go and Six Meter Walk, and flexibility with the Functional Reach Test. All physical exercise tests were used in non-pharmacological RCTs, except for the Tinetti Balance Scale that was also used in 1 pharmacological RCT.

Dementia Type

A majority of the participants were diagnosed with $\mathrm{AD}(84 \%)$ or $\mathrm{VaD}(6 \%)$. Six exercise tests were used only in $\mathrm{AD}$ patients and covered the physical exercise domains endurance capacity (Two Minute Step Test), muscle strength (30 Second Chair Stand), balance (Tinetti Balance Scale, Abnormal One-Leg Balance), and mobility (Timed Up and Go, Six Meter Walk). Physical exercise tests were not used in RCTs only including VaD patients. In RCTs that included both $\mathrm{AD}$ and $\mathrm{VaD}$ patients, 3 physical exercise tests measured the physical exercise domains endurance capacity (Six Minute Walk Distance), flexibility (Functional Reach Test), and balance (Berg Balance Scale).

Study Quality

The study quality of RCTs ranged from 5 (moderate) to 9 (excellent). Only the Tinetti Balance Scale was used in a RCT with excellent study quality (PEDro 9).

\section{Effect Size}

Pooled ESs of RCTs ranged from small $(d=0.02)$ to large $(d=0.87)$. A large ES was found with the Tinetti Balance Scale $(d=0.87)$. Medium ESs were found with the Six Meter Walk $(d=0.58)$ and the Six Minute Walk Distance $(d=0.51)$. 
Pers et al.: Recommended Measures for the Assessment of Cognitive and Physical

Performance in Older Patients with Dementia: A Systematic Review

Table 5. Domain-specific physical exercise tests, ordered on the basis of frequency of tests use, ES, study quality, reliability, and validity for the physical exercise domains endurance capacity, muscle strength, balance, and mobility

\begin{tabular}{lllll}
\hline & Endurance capacity & Muscle strength* & Balance & Mobility \\
\hline 1 & Six Minute Walk Distance $^{*, a-c}$ & Five Times Sit to Stand & Tinetti Balance Scale*, a, b & Timed Up and Go*, b, c $^{*}$ \\
\hline 2 & & 30 Second Chair Stand & & Six Meter Walk ${ }^{*}$ a-c \\
\hline
\end{tabular}

a Able to measure an effect (sensitivity to change). ${ }^{\mathrm{b}}$ Test was used in excellent/good-quality RCTs (PEDro). ${ }^{\mathrm{c}}$ Reliable/ valid in dementia patients. ${ }^{*}$ More research is needed to recommend these tests.

Reliability and Validity

The Timed Up and Go [intraclass correlation (ICC) $=0.985-0.988$ ], Six Minute Walk Distance $(\mathrm{ICC}=0.982-0.987)$, and Six Meter Walk $(\mathrm{ICC}=0.973-0.977)$ showed excellent test-retest values for older patients with dementia [153]. For the remaining physical exercise tests that are presented in table 3, no psychometric studies for the reliability and validity with dementia patients were found.

\section{Discussion}

To improve the study quality and increase comparability of clinical trials and observational studies, researchers should strive to use a limited number of generally accepted, feasible, reliable, and valid tests that cover the domains of cognitive and physical functioning. Following previous studies that recommended neuropsychological tests for the diagnoses of dementia $[20,21]$ and studies that stated the importance of physical exercise to attenuate cognitive impairment in older patients with dementia [154], the aim of the current review was to give up-to-date recommendations of both neuropsychological and physical exercise tests for high-quality experimental research with older patients with dementia.

\section{Neuropsychological Tests}

This study revealed 59 different neuropsychological tests that were used in 63 RCTs. This confirms the assumption that there is a large heterogeneity in neuropsychological test use in RCTs with older patients with dementia. The results showed that global cognitive tests were used more often in comparison with neuropsychological tests that measured one specific cognitive domain.

In particular, the global cognitive tests MMSE, ADAS-cog, and the SIB were standing out because of their excellent reliability and validity (table 2), and were often used in highquality RCTs which suggest that they are feasible. However, for all 3 tests the sensitivity to change was low. In line with this, the sensitivity to change of the MMSE and ADAS-cog was also challenged in other studies [140], because changes in performance measured with these tests can easily be caused by small measurement errors [125]. For the SIB, research [140] showed that this test is sensitive to change in patients with severe dementia. Altogether, on the basis of feasibility, sensitivity, reliability, and validity we recommend the use of the SIB in RCTs to measure global cognitive treatment effects.

Memory tests could not be selected in this review due to the large heterogeneity that was found in memory test use. Earlier work on memory tests for diagnosing dementia showed that verbal memory, visual memory, and non-verbal memory can be assessed with several tests [20]. Most of these tests were only used once in RCTs between 2005 and 2011, and 1 test 
Performance in Older Patients with Dementia: A Systematic Review

(Word List of the Consortium to Establish a Registry for Alzheimer's disease) was not used at all in RCTs over that period. Additionally, because these tests were specifically recommended for diagnosis of dementia, we suggest that it is not feasible to measure effects over time with these tests. Furthermore, studies that investigated the psychometric properties of these memory tests are lacking.

To measure executive functioning, we recommend the use of the Verbal Fluency Test Category, Clock Drawing Test, Verbal Fluency Test Letters, and the Trail Making Test-B because they were frequently used in good- or excellent-quality RCTs. However, we found that only the Verbal Fluency Test Category was able to detect change, and only the Clock Drawing Test was found to be reliable for the population of older dementia patients (table 2). Since information and psychometric quality is in many cases still insufficient, the recommended selection should be used with care and further evaluation of these tests is needed.

For attention we recommend the Digit Span Forward, Digit Span Backward, and Trail Making Test-A because of their frequent use in high-quality studies. However, no studies were found that investigated the psychometric properties of these tests. Furthermore, the results showed that the sensitivity to change was small.

Table 4 sums up the best currently available tests used in international intervention studies with older persons with dementia. Although they are widely applied, it was shown that the recommended neuropsychological tests lack psychometric studies. Therefore, future research into the psychometric quality of the tests found in this review is essential. The recommended selection of currently optimal cognitive tests should be used with care. Researchers are advised to select the recommended tests that most closely fit their study objectives.

\section{Physical Exercise Tests}

This review found 10 different exercise tests that covered the domains endurance capacity, muscle strength, balance, and mobility. However, there is a large heterogeneity in tests used and none of the tests were used frequently enough in RCTs to recommend them. Preliminary recommendations based on the results of this review may be a first step for the selection of exercise tests.

For endurance capacity, the results showed that the Six Minute Walk Distance is reliable [153] and sensitive to change. Muscle strength was measured with the Five Times Sit to Stand and the 30 Second Chair Stand. However, no studies were available that investigated the feasibility and psychometric properties for these tests. For balance, results showed that the Tinetti Balance Scale was sensitive to change, but again no studies were available that investigated the feasibility and psychometric properties. Mobility was measured with the Six Meter Walk and the Timed Up and Go. The results showed that both tests are reliable [153]. However, only the Six Meter Walk was sensitive to change. Based on the limited information at hand, the best exercise tests available so far are summed up in table 5.

Because of the importance of physical functioning in the disease process of dementia [154], it is essential that future research obtains more information on the feasibility, sensitivity to change, reliability, and validity of physical exercise tests that were found in this review. Since this information is in many cases still insufficient, the recommendation of optimal physical tests should be used with care.

\section{Conclusion}

This review mapped the large heterogeneity in cognitive and physical functioning tests used in international intervention studies with older persons with dementia. The provided neuropsychological (table 4) and exercise (table 5) test recommendations from this system- 
Bossers et al.: Recommended Measures for the Assessment of Cognitive and Physical

Performance in Older Patients with Dementia: A Systematic Review

atic analysis may lead to a more evidence-based choice of tests that better fit the research questions of future studies. Since information on psychometric quality is in many cases still insufficient, the recommended selection of currently optimal cognitive and physical tests should be used with care. Researchers are advised to select those recommended tests that most closely fit their study objectives.

\section{Disclosure Statement}

Open access costs were funded by Fonds NutsOhra. All authors declare that there are no conflicts of interest.

\section{References}

-1 Ferri CP, Prince M, Brayne C, Brodaty H, Fratiglioni L, Ganguli M, Hall K, Hasegawa K, Hendrie H, Huang Y, Jorm A, Mathers C, Menezes PR, Rimmer E, Scazufca M, Alzheimer's Disease International: Global prevalence of dementia: a Delphi consensus study. Lancet 2005;366:2112-2117.

-2 Whitehouse PJ, Price DL, Struble RG, Clark AW, Coyle JT, Delon MR: Alzheimer's disease and senile dementia: loss of neurons in the basal forebrain. Science 1982;215:1237-1239.

-3 Thomas VS, Hageman PA: Can neuromuscular strength and function in people with dementia be rehabilitated using resistance-exercise training? Results from a preliminary intervention study. J Gerontol A Biol Sci Med Sci 2003;58:746-751.

-4 Clark CM, Forman MS: Frontotemporal lobar degeneration with motor neuron disease: a clinical and pathological spectrum. Arch Neurol 2006;63:489-490.

5 Bird TD: Genetic aspects of Alzheimer disease. Genet Med 2008;10:231-239.

-6 Barberger-Gateau P, Fabrigoule C, Amieva H, Helmer C, Dartigues JF: The disablement process: a conceptual framework for dementia-associated disability. Dement Geriatr Cogn Disord 2002;13: $60-66$.

-7 Gershon RC, Cella D, Fox NA, Havlik RJ, Hendrie HC, Wagster MV: Assessment of neurological and behavioural function: the NIH toolbox. Lancet Neurol 2010;9:138-139.

8 Bruce B, Fries JF, Ambrosini D, Lingala B, Gandek B, Rose M, Ware JE Jr: Better assessment of physical function: item improvement is neglected but essential. Arthritis Res Ther 2009;11:R191.

-9 Sauvaget C, Yamada M, Fujiwara S, Sasaki H, Mimori Y: Dementia as a predictor of functional disability: a four-year follow-up study. Gerontology 2002;48:226-233.

-10 Traykov L, Rigaud AS, Cesaro P, Boller F: Neuropsychological impairment in the early Alzheimer's disease. Encephale 2007;33:310-316.

-11 Haan MN, Wallace R: Can dementia be prevented? Brain aging in a population-based context. Annu Rev Public Health 2004;25:1-24.

-12 Heyn P, Abreu BC, Ottenbacher KJ: The effects of exercise training on elderly persons with cognitive impairment and dementia: a meta-analysis. Arch Phys Med Rehabil 2004;85:1694-1704.

-13 Eggermont L, Swaab D, Luiten P, Scherder E: Exercise, cognition and Alzheimer's disease: more is not necessarily better. Neurosci Biobehav Rev 2006;30:562-575.

-14 Yu F, Kolanowski AM, Strumpf NE, Eslinger PJ: Improving cognition and function through exercise intervention in Alzheimer's disease. J Nurs Scholarsh 2006;38:358-365.

-15 Netz Y, Axelrad S, Argov E: Group physical activity for demented older adults: feasibility and effectiveness. Clin Rehabil 2007;21:977-986.

16 Forbes D, Forbes S, Morgan DG, Markle-Reid M, Wood J, Culum I: Physical activity programs for persons with dementia. Cochrane Database Syst Rev 2008;3:CD006489.

-17 Liu-Ambrose T, Donaldson MG: Exercise and cognition in older adults: is there a role for resistance training programmes? Br J Sports Med 2009;43:25-27.

-18 Mangialasche F, Solomon A, Winblad B, Mecocci P, Kivipelto M: Alzheimer’s disease: clinical trials and drug development. Lancet Neurol 2010;9:702-716. 
19 Maruta C, Guerreiro M, de Mendonca A, Hort J, Scheltens P: The use of neuropsychological tests across Europe: the need for a consensus in the use of assessment tools for dementia. Eur J Neurol 2011;18:279-285.

20 Chaves MLF, Godinho CC, Porto CS, Mansur L, Carthery-Goulart MT, Yassuda MS, Beato R: Cognitive, functional and behavioral assessment: Alzheimer's disease. Dement Neuropsychol 2011;5: 153-166.

21 Young J, Meagher D, Maclullich A: Cognitive assessment of older people. BMJ 2011;343:d5042.

22 Cohen J: Statistical Power Analysis for the Behavioural Sciences, ed 2. Hillsdale, Erlbaum, 1988.

23 Blankevoort CG, van Heuvelen MJ, Boersma F, Luning H, de Jong J, Scherder EJ: Review of effects of physical activity on strength, balance, mobility and ADL performance in elderly subjects with dementia. Dement Geriatr Cogn Disord 2010;30:392-402.

24 Thalheimer W, Cook S: How to calculate effect sizes from published research articles: a simplified methodology. 2002. http://worl-learning.com/effect_sizes.htm (accessed June 8, 2011).

25 De Morton NA: The PEDro scale is a valid measure of the methodological quality of clinical trials: a demographic study. Aust J Physiother 2009;55:129-133.

26 Aisen PS, Saumier D, Briand R, Laurin J, Gervais F, Tremblay P, Garceau D: A phase II study targeting amyloid-beta with 3APS in mild-to-moderate Alzheimer disease. Neurology 2006;67:1757-1763.

27 Alvarez XA, Cacabelos R, Laredo M, Couceiro V, Sampedro C, Varela M, Corzo L, Fernandez-Novoa L, Vargas M, Aleixandre M, Linares C, Granizo E, Muresanu D, Moessler H: A 24-week, doubleblind, placebo-controlled study of three dosages of cerebrolysin in patients with mild to moderate Alzheimer's disease. Eur J Neurol 2006;13:43-54.

28 Avila R, Carvalho IA, Bottino CM, Miotto EC: Neuropsychological rehabilitation in mild and moderate Alzheimer's disease patients. Behav Neurol 2007;18:225-233.

29 Bentham P, Gray R, Sellwood E, Hills R, Crome P, Raftery J: Aspirin in Alzheimer's disease (AD2000): a randomised open-label trial. Lancet Neurol 2008;7:41-49.

30 Black SE, Doody R, Li H, McRae T, Jambor KM, Xu Y, Sun Y, Perdomo CA, Richardson S: Donepezil preserves cognition and global function in patients with severe Alzheimer disease. Neurology 2007; 69:459-469.

31 Bottino CMC, Carvalho IAM, Alvarez AMMA, Avila R, Zukauskas PR, Bustamante SEZ, Andrade FC, Hototian SR, Saffi F, Camargo CHP: Cognitive rehabilitation combined with drug treatment in Alzheimer's disease patients: a pilot study. Clin Rehabil 2005;19:861-869.

32 Boxer AL, Lipton AM, Womack K, Merrilees J, Neuhaus J, Pavlic D, Gandhi A, Red D, Martin-Cook K, Svetlik D, Miller BL: An open-label study of memantine treatment in 3 subtypes of frontotemporal lobar degeneration. Alzheimer Dis Assoc Disord 2009;23:211-217.

33 Cheng ST, Chan AC, Yu EC: An exploratory study of the effect of mahjong on the cognitive functioning of persons with dementia. Int J Geriatr Psychiatry 2006;21:611-617.

34 Choi AN, Lee MS, Cheong KJ, Lee JS: Effects of group music intervention on behavioral and psychological symptoms in patients with dementia: a pilot-controlled trial. Int J Neurosci 2009;119:471-481.

35 Cummings JL, Koumaras B, Chen M, Mirski D: Effects of rivastigmine treatment on the neuropsychiatric and behavioral disturbances of nursing home residents with moderate to severe probable Alzheimer's disease: a 26-week, multicenter, open-label study. Am J Geriatr Pharmacother 2005;3: $137-148$

36 Farina E, Mantovani F, Fioravanti R, Pignatti R, Chiavari L, Imbornone E, Olivotto F, Alberoni M, Mariani C, Nemni R: Evaluating two group programmes of cognitive training in mild-to-moderate $\mathrm{AD}$ : is there any difference between a 'global' stimulation and a 'cognitive-specific' one? Aging Ment Health 2006;10:211-218.

37 Feldman H, Gauthier S, Hecker J, Vellas B, Xu Y, Ieni JR, Schwam EM: Efficacy and safety of donepezil in patients with more severe Alzheimer's disease: a subgroup analysis from a randomized, placebo-controlled trial. Int J Geriatr Psychiatry 2005;20:559-569.

38 Frankfort SV, Appels BA, de BA, Tulner LR, van Campen JP, Koks CH, Beijnen JH, Schmand BA: Identification of responders and reactive domains to rivastigmine in Alzheimer's disease. Pharmacoepidemiol Drug Saf 2007; 16:545-551.

39 Hampel H, Ewers M, Burger K, Annas P, Mortberg A, Bogstedt A, Frolich L, Schroder J, Schonknecht P, Riepe MW, Kraft I, Gasser T, Leyhe T, Moller HJ, Kurz A, Basun H: Lithium trial in Alzheimer's disease: a randomized, single-blind, placebo-controlled, multicenter 10-week study. J Clin Psychiatry 2009;70:922-931. 
40 Ito T, Meguro K, Akanuma K, Ishii H, Mori E: A randomized controlled trial of the group reminiscence approach in patients with vascular dementia. Dement Geriatr Cogn Disord 2007;24:48-54.

41 Johannsen P, Salmon E, Hampel H, Xu Y, Richardson S, Qvitzau S, Schindler R: Assessing therapeutic efficacy in a progressive disease: a study of donepezil in Alzheimer's disease. CNS Drugs 2006;20: $311-325$.

42 Kadir A, Andreasen N, Almkvist O, Wall A, Forsberg A, Engler H, Hagman G, Larksater M, Winblad B, Zetterberg H, Blennow K, Langstrom B, Nordberg A: Effect of phenserine treatment on brain functional activity and amyloid in Alzheimer's disease. Ann Neurol 2008;63:621-631.

43 Kennedy J, Deberdt W, Siegal A, Micca J, Degenhardt E, Ahl J, Meyers A, Kaiser C, Baker RW: Olanzapine does not enhance cognition in non-agitated and non-psychotic patients with mild to moderate Alzheimer's dementia. Int J Geriatr Psychiatry 2005;20:1020-1027.

44 Kurlan R, Cummings J, Raman R, Thal L: Quetiapine for agitation or psychosis in patients with dementia and parkinsonism. Neurology 2007;68:1356-1363.

45 Kwak YS, Um SY, Son TG, Kim DJ: Effect of regular exercise on senile dementia patients. Int J Sports Med 2008;29:471-474.

46 Kwok T, Lee J, Lam L, Woo J: Vitamin B(12) supplementation did not improve cognition but reduced delirium in demented patients with vitamin B(12) deficiency. Arch Gerontol Geriatr 2008;46:273282.

47 Lannfelt L, Blennow K, Zetterberg H, Batsman S, Ames D, Harrison J, Masters CL, Targum S, Bush AI, Murdoch R, Wilson J, Ritchie CW: Safety, efficacy, and biomarker findings of PBT2 in targeting $\mathrm{A}$ (beta) as a modifying therapy for Alzheimer's disease: a phase IIa, double-blind, randomised, placebo-controlled trial. Lancet Neurol 2008;7:779-786.

48 Lee ST, Chu K, Sim JY, Heo JH, Kim M: Panax ginseng enhances cognitive performance in Alzheimer disease. Alzheimer Dis Assoc Disord 2008;22:222-226.

49 Lee SB, Park CS, Jeong JW, Choe JY, Hwang YJ, Park CA, Park JH, Lee DY, Jhoo JH, Kim KW: Effects of spaced retrieval training (SRT) on cognitive function in Alzheimer's disease (AD) patients. Arch Gerontol Geriatr 2009;49:289-293.

50 Levin OS, Batukaeva LA, Smolentseva IG, Amosova NA: Efficacy and safety of memantine in Lewy body dementia. Neurosci Behav Physiol 2009;39:597-604.

51 Winblad B, Wimo A, Engedal K, Soininen H, Verhey F, Waldemar G, Wetterholm AL, Haglund A, Zhang R, Schindler R: 3-year study of donepezil therapy in Alzheimer's disease: effects of early and continuous therapy. Dement Geriatr Cogn Disord 2006;21:353-363.

52 Mazza M, Capuano A, Bria P, Mazza S: Ginkgo biloba and donepezil: a comparison in the treatment of Alzheimer's dementia in a randomized placebo-controlled double-blind study. Eur J Neurol 2006; 13:981-985.

53 Mizukami K, Asada T, Kinoshita T, Tanaka K, Sonohara K, Nakai R, Yamaguchi K, Hanyu H, Kanaya K, Takao T, Okada M, Kudo S, Kotoku H, Iwakiri M, Kurita H, Miyamura T, Kawasaki Y, Omori K, Shiozaki K, Odawara T, Suzuki T, Yamada S, Nakamura Y, Toba K: A randomized cross-over study of a traditional Japanese medicine (kampo), yokukansan, in the treatment of the behavioural and psychological symptoms of dementia. Int J Neuropsychopharmacol 2009;12:191-199.

54 Modrego PJ, Pina MA, Fayed N, Diaz M: Changes in metabolite ratios after treatment with rivastigmine in Alzheimer's disease: a nonrandomised controlled trial with magnetic resonance spectroscopy. CNS Drugs 2006;20:867-877.

55 Mohs RC, Shiovitz TM, Tariot PN, Porsteinsson AP, Baker KD, Feldman PD: Atomoxetine augmentation of cholinesterase inhibitor therapy in patients with Alzheimer disease: 6-month, randomized, double-blind, placebo-controlled, parallel-trial study. Am J Geriatr Psychiatry 2009;17:752-759.

56 Moretti R, Torre P, Antonello RM, Cazzato G, Pizzolato G: Different responses to rivastigmine in subcortical vascular dementia and multi-infarct dementia. Am J Alzheimers Dis Other Demen 2008; 23:167-176.

57 Mowla A, Pani A: Comparison of topiramate and risperidone for the treatment of behavioral disturbances of patients with Alzheimer disease: a double-blind, randomized clinical trial. J Clin Psychopharmacol 2010;30:40-43.

58 Onder G, Zanetti O, Giacobini E, Frisoni GB, Bartorelli L, Carbone G, Lambertucci P, Silveri MC, Bernabei R: Reality orientation therapy combined with cholinesterase inhibitors in Alzheimer's disease: randomised controlled trial. Br J Psychiatry 2005;187:450-455. 
59 Onor ML, Trevisiol M, Negro C, Signorini A, Saina M, Aguglia E: Impact of a multimodal rehabilitative intervention on demented patients and their caregivers. Am J Alzheimers Dis Other Demen 2007;22:261-272.

60 Ootani M, Nara I, Kaneko F, Okamura H: Construction of a speed feedback therapy system to improve cognitive impairment in elderly people with dementia: a preliminary report. Dement Geriatr Cogn Disord 2005;20:105-111.

61 Penner J, Rupsingh R, Smith M, Wells JL, Borrie MJ, Bartha R: Increased glutamate in the hippocampus after galantamine treatment for Alzheimer disease. Prog Neuropsychopharmacol Biol Psychiatry 2010;34:104-110.

62 Plastino M, Fava A, Pirritano D, Cotronei P, Sacco N, Sperli T, Spano A, Gallo D, Mungari P, Consoli D, Bosco D: Effects of insulinic therapy on cognitive impairment in patients with Alzheimer disease and diabetes mellitus type-2. J Neurol Sci 2010;288:112-116.

63 Porsteinsson AP, Grossberg GT, Mintzer J, Olin JT: Memantine treatment in patients with mild to moderate Alzheimer's disease already receiving a cholinesterase inhibitor: a randomized, doubleblind, placebo-controlled trial. Curr Alzheimer Res 2008;5:83-89.

64 Potkin SG, Alva G, Gunay I, Koumaras B, Chen M, Marski D: A pilot study evaluating the efficacy and safety of rivastigmine in patients with mixed dementia. Drugs Aging 2006;23:241-249.

65 Rainer M, Haushofer M, Pfolz H, Struhal C, Wick W: Quetiapine versus risperidone in elderly patients with behavioural and psychological symptoms of dementia: efficacy, safety and cognitive function. Eur Psychiatry 2007;22:395-403.

66 Reisberg B, Doody R, Stoffler A, Schmitt F, Ferris S, Mobius HJ: A 24-week open-label extension study of memantine in moderate to severe Alzheimer disease. Arch Neurol 2006;63:49-54.

67 Requena C, Maestu F, Campo P, Fernandez A, Ortiz T: Effects of cholinergic drugs and cognitive training on dementia: 2-year follow-up. Dement Geriatr Cogn Disord 2006;22:339-345.

68 Roman GC, Wilkinson DG, Doody RS, Black SE, Salloway SP, Schindler RJ: Donepezil in vascular dementia: combined analysis of two large-scale clinical trials. Dement Geriatr Cogn Disord 2005;20: $338-344$.

69 Savaskan E, Schnitzler C, Schroder C, Cajochen C, Muller-Spahn F, Wirz-Justice A: Treatment of behavioural, cognitive and circadian rest-activity cycle disturbances in Alzheimer's disease: haloperidol vs. quetiapine. Int J Neuropsychopharmacol 2006;9:507-516.

70 Scheltens P, Kamphuis PJ, Verhey FR, Olde Rikkert MG, Wurtman RJ, Wilkinson D, Twisk JW, Kurz A: Efficacy of a medical food in mild Alzheimer's disease: a randomized, controlled trial. Alzheimers Dement 2010;6:1-10.

71 Soininen H, West C, Robbins J, Niculescu L: Long-term efficacy and safety of celecoxib in Alzheimer's disease. Dement Geriatr Cogn Disord 2007;23:8-21.

72 Sparks DL, Connor DJ, Sabbagh MN, Petersen RB, Lopez J, Browne P: Circulating cholesterol levels, apolipoprotein $\mathrm{E}$ genotype and dementia severity influence the benefit of atorvastatin treatment in Alzheimer's disease: results of the Alzheimer's Disease Cholesterol-Lowering Treatment (ADCLT) trial. Acta Neurol Scand Suppl 2006;185:3-7.

73 Bullock R, Bergman H, Touchon J, Gambina G, He Y, Nagel J, Lane R: Effect of age on response to rivastigmine or donepezil in patients with Alzheimer's disease. Curr Med Res Opin 2006;22:483494.

74 Winblad B, Kilander L, Eriksson S, Minthon L, Batsman S, Wetterholm AL, Jansson-Blixt C, Haglund A, Severe Alzheimer's Disease Study Group: Donepezil in patients with severe Alzheimer's disease: double-blind, parallel-group, placebo-controlled study. Lancet 2006;367:1057-1065.

75 Winstein CJ, Bentzen KR, Boyd L, Schneider LS: Does the cholinesterase inhibitor, donepezil, benefit both declarative and non-declarative processes in mild to moderate Alzheimer's disease? Curr Alzheimer Res 2007;4:273-276.

76 Yu J, Zhang X, Liu C, Meng Y, Han J: Effect of acupuncture treatment on vascular dementia. Neurol Res 2006;28:97-103.

77 Miu DKY, Szeto SL, Mak YF: A randomized controlled trial on the effect of exercise on physical, cognitive and affective function in dementia subjects. Asian J Gerontol Geriatr 2008;3:8-16.

78 Yaguez L, Shaw KN, Morris R, Matthews D: The effects on cognitive functions of a movement-based intervention in patients with Alzheimer's type dementia: a pilot study. Int J Geriatr Psychiatry 2011; 26:173-181. 
79 Venturelli M, Scarsini R, Schena F: Six-month walking program changes cognitive and ADL performance in patients with Alzheimer. Am J Alzheimers Dis Other Demen 2011;26:381-388.

80 Aronson S, Van Baelen B, Kavanagh S, Schwalen S: Optimal dosing of galantamine in patients with mild or moderate Alzheimer's disease: post hoc analysis of a randomized, double-blind, placebocontrolled trial. Drugs Aging 2009;26:231-239.

81 Auchus AP, Brashear HR, Salloway S, Korczyn AD, De Deyn PP, Gassmann-Mayer C: Galantamine treatment of vascular dementia: a randomized trial. Neurology 2007;69:448-458.

82 Bakchine S, Loft H: Memantine treatment in patients with mild to moderate Alzheimer's disease: results of a randomised, double-blind, placebo-controlled 6-month study. J Alzheimers Dis 2007;11: 471-479.

83 Chappell AS, Gonzales C, Williams J, Witte MM, Mohs RC, Sperling R: AMPA potentiator treatment of cognitive deficits in Alzheimer disease. Neurology 2007;68:1008-1012.

84 Lu PH, Masterman DA, Mulnard R, Cotman C, Miller B, Yaffe K, Reback E, Porter V, Swerdloff R, Cummings JL: Effects of testosterone on cognition and mood in male patients with mild Alzheimer disease and healthy elderly men. Arch Neurol 2006;63:177-185.

85 Modrego PJ, Fayed N, Errea JM, Rios C, Pina MA, Sarasa M: Memantine versus donepezil in mild to moderate Alzheimer's disease: a randomized trial with magnetic resonance spectroscopy. Eur J Neurol 2010;17:405-412.

86 Mori S, Mori E, Iseki E, Kosaka K: Efficacy and safety of donepezil in patients with dementia with Lewy bodies: preliminary findings from an open-label study. Psychiatry Clin Neurosci 2006;60: 190-195.

87 Muresanu DF, Alvarez XA, Moessler H, Buia M, Stan A, Pintea D, Moldovan F, Popescu BO: A pilot study to evaluate the effects of cerebrolysin on cognition and $\mathrm{qEEG}$ in vascular dementia: cognitive improvement correlates with qEEG acceleration. J Neurol Sci 2008;267:112-119.

88 Paleacu D, Barak Y, Mirecky I, Mazeh D: Quetiapine treatment for behavioural and psychological symptoms of dementia in Alzheimer's disease patients: a 6-week, double-blind, placebo-controlled study. Int J Geriatr Psychiatry 2008;23:393-400.

89 Peskind ER, Potkin SG, Pomara N, Ott BR, Graham SM, Olin JT, McDonald S: Memantine treatment in mild to moderate Alzheimer disease: a 24-week randomized, controlled trial. Am J Geriatr Psychiatry 2006;14:704-715.

90 Pomara N, Ott BR, Peskind E, Resnick EM: Memantine treatment of cognitive symptoms in mild to moderate Alzheimer disease: secondary analyses from a placebo-controlled randomized trial. Alzheimer Dis Assoc Disord 2007;21:60-64.

91 Raggi A, Iannaccone S, Marcone A, Ginex V, Ortelli P, Nonis A, Giusti MC, Cappa SF: The effects of a comprehensive rehabilitation program of Alzheimer's disease in a hospital setting. Behav Neurol 2007;18:1-6.

92 Salloway S, Sperling R, Gilman S, Fox NC, Blennow K, Raskind M, Sabbagh M, Honig LS, Doody R, van Dyck CH, Mulnard R, Barakos J, Gregg KM, Liu E, Lieberburg I, Schenk D, Black R, Grundman M: A phase 2 multiple ascending dose trial of bapineuzumab in mild to moderate Alzheimer disease. Neurology 2009;73:2061-2070.

93 Suh GH, Jung HY, Lee CU, Oh BH, Lee SK, Lee N, Kim J, Kee BS, Ko D, Kim YH, Ju YS, Hong I, Choi $S$ : Effect of the apolipoprotein E epsilon4 allele on the efficacy and tolerability of galantamine in the treatment of Alzheimer's disease. Dement Geriatr Cogn Disord 2006;21:33-39.

94 Wilkinson D, Schindler R, Schwam E, Waldemar G, Jones RW, Gauthier S, Lopez OL, Cummings J, $\mathrm{Xu}$ Y, Feldman HH: Effectiveness of donepezil in reducing clinical worsening in patients with mildto-moderate Alzheimer's disease. Dement Geriatr Cogn Disord 2009;28:244-251.

95 Winblad B, Grossberg G, Frolich L, Farlow M, Zechner S, Nagel J, Lane R: IDEAL: a 6-month, doubleblind, placebo-controlled study of the first skin patch for Alzheimer disease. Neurology 2007;69:S14S22.

96 McCarney R, Fisher P, Iliffe S, van Haselen R, Griffin M, van der Meulen J, Warner J: Ginkgo biloba for mild to moderate dementia in a community setting: a pragmatic, randomised, parallel-group, double-blind, placebo-controlled trial. Int J Geriatr Psychiatry 2008;23:1222-1230.

97 Frolich L, Ashwood T, Nilsson J, Eckerwall G, Sirocco Investigators: Effects of AZD3480 on cognition in patients with mild-to-moderate Alzheimer's disease: a phase IIb dose-finding study. J Alzheimers Dis 2011;24:363-374. 
98 Cherrier MM, Matsumoto AM, Amory JK, Asthana S, Bremner W, Peskind ER, Raskind MA, Craft S: Testosterone improves spatial memory in men with Alzheimer disease and mild cognitive impairment. Neurology 2005;64:2063-2068.

99 Eggermont LH, Swaab DF, Hol EM, Scherder EJ: Walking the line: a randomised trial on the effects of a short term walking programme on cognition in dementia. J Neurol Neurosurg Psychiatry 2009; 80:802-804.

100 Napryeyenko O, Sonnik G, Tartakovsky I: Efficacy and tolerability of ginkgo biloba extract EGb 761 by type of dementia: analyses of a randomised controlled trial. J Neurol Sci 2009;283:224-229.

101 Ancoli-Israel S, Palmer BW, Cooke JR, Corey-Bloom J, Fiorentino L, Natarajan L, Liu L, Ayalon L, He F, Loredo JS: Cognitive effects of treating obstructive sleep apnea in Alzheimer's disease: a randomized controlled study. J Am Geriatr Soc 2008;56:2076-2081.

102 Scherder EJ, van Tol MJ, Swaab DF: High-frequency cranial electrostimulation (CES) in patients with probable Alzheimer's disease. Am J Phys Med Rehabil 2006;85:614-618.

103 Scherder EJ, Vuijk PJ, Swaab DF, Van Someren EJ: Estimating the effects of right median nerve stimulation on memory in Alzheimer's disease: a randomized controlled pilot study. Exp Aging Res 2007; 33:177-186.

104 Eggermont LH, Knol DL, Hol EM, Swaab DF, Scherder EJ: Hand motor activity, cognition, mood, and the rest-activity rhythm in dementia A clustered RCT. Behav Brain Res 2009;196:271-278.

105 Homma A, Imai Y, Tago H, Asada T, Shigeta M, Iwamoto T, Takita M, Arimoto I, Koma H, Ohbayashi T: Donepezil treatment of patients with severe Alzheimer's disease in a Japanese population: results from a 24-week, double-blind, placebo-controlled, randomized trial. Dement Geriatr Cogn Disord 2008;25:399-407.

106 Schmitt FA, van Dyck CH, Wichems CH, Olin JT: Cognitive response to memantine in moderate to severe Alzheimer disease patients already receiving donepezil: an exploratory reanalysis. Alzheimer Dis Assoc Disord 2006;20:255-262.

107 van Dyck CH, Schmitt FA, Olin JT: A responder analysis of memantine treatment in patients with Alzheimer disease maintained on donepezil. Am J Geriatr Psychiatry 2006;14:428-437.

108 Stevens J, Killeen M: A randomised controlled trial testing the impact of exercise on cognitive symptoms and disability of residents with dementia. Contemp Nurse 2006;21:32-40.

109 Rahman S, Robbins TW, Hodges JR, Mehta MA, Nestor PJ, Clark L, Sahakian BJ: Methylphenidate ('ritalin') can ameliorate abnormal risk-taking behavior in the frontal variant of frontotemporal dementia. Neuropsychopharmacology 2006;31:651-658.

110 Paskavitz JF, Gunstad JJ, Samuel JE: Clock drawing and frontal lobe behavioral effects of memantine in Alzheimer's disease: a rater-blinded study. Am J Alzheimers Dis Other Demen 2006;21:454-459.

111 Kemoun G, Thibaud M, Roumagne N, Carette P, Albinet C, Toussaint L, Paccalin M, Dugue B: Effects of a physical training programme on cognitive function and walking efficiency in elderly persons with dementia. Dement Geriatr Cogn Disord 2010;29:109-114.

112 Anthony JC, LeResche L, Niaz U, von Korff MR, Folstein MF: Limits of the 'Mini-Mental State' as a screening test for dementia and delirium among hospital patients. Psychol Med 1982;12:397-408.

113 Folstein MF, Folstein SE, McHugh PR: 'Mini-Mental State'. A practical method for grading the cognitive state of patients for the clinician. J Psychiatr Res 1975;12:189-198.

114 Dick JP, Guiloff RJ, Stewart A, Blackstock J, Bielawska C, Paul EA, Marsden CD: Mini-Mental State Examination in neurological patients. J Neurol Neurosurg Psychiatry 1984;47:496-499.

115 Molloy DW, Alemayehu E, Roberts R: Reliability of a Standardized Mini-Mental State Examination compared with the traditional Mini-Mental State Examination. Am J Psychiatry 1991;148:102-105.

116 Fillenbaum GG, Heyman A, Wilkinson WE, Haynes CS: Comparison of two screening tests in Alzheimer's disease. The correlation and reliability of the Mini-Mental State Examination and the Modified Blessed Test. Arch Neurol 1987;44:924-927.

117 Thal LJ, Grundman M, Golden R: Alzheimer's disease: a correlational analysis of the Blessed Information-Memory-Concentration Test and the Mini-Mental State Exam. Neurology 1986;36:262-264.

118 Uhlmann RF, Larson EB, Buchner DM: Correlations of Mini-Mental State and Modified Dementia Rating Scale to measures of transitional health status in dementia. J Gerontol 1987;42:33-36.

119 Tombaugh TN, McIntyre NJ: The Mini-Mental State Examination: a comprehensive review. J Am Geriatr Soc 1992;40:922-935. 
120 McDowell I, Kristjansson B, Hill GB, Hebert R: Community screening for dementia: the Mini Mental State Exam (MMSE) and Modified Mini-Mental State Exam (3MS) compared. J Clin Epidemiol 1997;50:377-383.

121 Holzer CE, Tischler GL, Leaf PJ, Myers JK: An epidemiologic assessment of cognitive impairment in a community population. Res Com Health 1984;4:3-32.

122 Kay DW, Henderson AS, Scott R, Wilson J, Rickwood D, Grayson DA: Dementia and depression among the elderly living in the Hobart community: the effect of the diagnostic criteria on the prevalence rates. Psychol Med 1985;15:771-788.

123 Foreman MD: Reliability and validity of mental status questionnaires in elderly hospitalized patients. Nurs Res 1987;36:216-220.

124 Albert M, Cohen C: The test for severe impairment: an instrument for the assessment of patients with severe cognitive dysfunction. J Am Geriatr Soc 1992;40:449-453.

125 Tombaugh TN, McDowell I, Kristjansson B, Hubley AM: Mini-Mental State Examination (MMSE) and the Modified MMSE (3MS): a psychometric comparison and normative data. Arch Clin Neuropsychol 1996;8:48-59.

126 Mavioglu H, Gedizlioglu M, Akyel S, Aslaner T, Eser E: The validity and reliability of the Turkish version of Alzheimer's Disease Assessment Scale-cognitive subscale (ADAS-cog) in patients with mild and moderate Alzheimer's disease and normal subjects. Int J Geriatr Psychiatry 2006;21:259265.

127 Rosen WG, Mohs RC, Davis KL: A new rating scale for Alzheimer’s disease. Am J Psychiatry 1984; 141:1356-1364.

128 Pena-Casanova J: Alzheimer's disease assessment scale - cognitive in clinical practice. Int Psychogeriatr 1997;9(Suppl 1):105-114.

129 Weyer G, Erzigkeit H, Kanowski S, Ihl R, Hadler D: Alzheimer's disease assessment scale: reliability and validity in a multicenter clinical trial. Int Psychogeriatr 1997;9:123-138.

130 Liu HC, Teng EL, Chuang YY, Lin KN, Fuh JL, Wang PN: The Alzheimer's disease assessment scale: findings from a low-education population. Dement Geriatr Cogn Disord 2002;13:21-26.

131 Chu LW, Chiu KC, Hui SL, Yu GK, Tsui WJ, Lee PW: The reliability and validity of the Alzheimer's Disease Assessment Scale cognitive subscale (ADAS-cog) among the elderly Chinese in Hong Kong. Ann Acad Med Singapore 2000;29:474-485.

132 Kim YS, Nibbelink DW, Overall JE: Factor structure and reliability of the Alzheimer's disease assessment scale in a multicenter trial with linopirdine. J Geriatr Psychiatry Neurol 1994;7:74-83.

133 Mohs RC: The Alzheimer's disease assessment scale. Int Psychogeriatr 1996;8:195-203.

134 Tsolaki M, Fountoulakis K, Nakopoulou E, Kazis A, Mohs RC: Alzheimer's disease assessment scale: the validation of the scale in Greece in elderly demented patients and normal subjects. Dement Geriatr Cogn Disord 1997;8:273-280.

135 Cano SJ, Posner HB, Moline ML, Hurt SW, Swartz J, Hsu T, Hobart JC: The ADAS-cog in Alzheimer's disease clinical trials: psychometric evaluation of the sum and its parts. J Neurol Neurosurg Psychiatry 2010;81:1363-1368.

136 Ahn IS, Kim JH, Ku HM, Saxton J, Kim DK: Reliability and validity of the severe impairment battery (SIB) in Korean dementia patients. J Korean Med Sci 2006;21:506-517.

137 Suh GH, Kang CJ: Validation of the severe impairment battery for patients with Alzheimer's disease in Korea. Int J Geriatr Psychiatry 2006;21:626-632.

138 Panisset M, Roudier M, Saxton J, Boller F: Severe impairment battery. A neuropsychological test for severely demented patients. Arch Neurol 1994;51:41-45.

139 Schmitt FA, Ashford W, Ernesto C, Saxton J, Schneider LS, Clark CM, Ferris SH, Mackell JA, Schafer $\mathrm{K}$, Thal LJ: The severe impairment battery: concurrent validity and the assessment of longitudinal change in Alzheimer's disease. The Alzheimer's Disease Cooperative Study. Alzheimer Dis Assoc Disord 1997;11(Suppl 2):S51-S56.

140 Bergh S, Selbaek G, Engedal K: Reliability and validity of the Norwegian version of the Severe Impairment Battery (SIB). Int J Geriatr Psychiatry 2008;23:896-902.

141 Schmitt FA, Saxton JA, Xu Y, McRae T, Sun Y, Richardson S, Li H: A brief instrument to assess treatment response in the patient with advanced Alzheimer disease. Alzheimer Dis Assoc Disord 2009; 23:377-383.

$142 \mathrm{Kim} \mathrm{H}$, Chey J: Effects of education, literacy, and dementia on the Clock Drawing Test performance. J Int Neuropsychol Soc 2010;16:1138-1146. 
143 Shulman KI, Pushkar Gold D, Cohen CA, Zucchero CA: Clock-drawing and dementia in the community: a longitudinal study. Int J Geriatr Psychiatry 1993;8:487-496.

144 Sunderland T, Hill JL, Mellow AM, Lawlor BA, Gundersheimer J, Newhouse PA, Grafman JH: Clock drawing in Alzheimer's disease. A novel measure of dementia severity. J Am Geriatr Soc 1989;37: $725-729$.

145 Wolf-Klein GP, Silverstone FA, Levy AP, Brod MS: Screening for Alzheimer's disease by clock drawing. J Am Geriatr Soc 1989;37:730-734.

146 Schramm U, Berger G, Muller R, Kratzsch T, Peters J, Frolich L: Psychometric properties of Clock Drawing Test and MMSE or Short Performance Test (SKT) in dementia screening in a memory clinic population. Int J Geriatr Psychiatry 2002;17:254-260.

147 Manos PJ: The utility of the Ten-Point Clock Test as a screen for cognitive impairment in general hospital patients. Gen Hosp Psychiatry 1997;19:439-444.

148 Chiu YC, Li CL, Lin KN, Chiu YF, Liu HC: Sensitivity and specificity of the Clock Drawing Test, incorporating Rouleau scoring system, as a screening instrument for questionable and mild dementia: scale development. Int J Nurs Stud 2008;45:75-84.

149 Seigerschmidt E, Mosch E, Siemen M, Forstl H, Bickel H: The Clock Drawing Test and questionable dementia: reliability and validity. Int J Geriatr Psychiatry 2002;17:1048-1054.

150 Netz Y, Axelrad S, Argov E: Group physical activity for demented older adults - feasibility and effectiveness. Clin Rehabil 2007;21:977-986.

151 Rolland Y, Pillard F, Klapouszczak A, Reynish E, Thomas D, Andrieu S, Riviere D, Vellas B: Exercise program for nursing home residents with Alzheimer's disease: a 1-year randomized, controlled trial. J Am Geriatr Soc 2007;55:158-165.

152 Santana-Sosa E, Barriopedro MI, Lopez-Mojares LM, Perez M, Lucia A: Exercise training is beneficial for Alzheimer's patients. Int J Sports Med 2008;29:845-850.

153 Ries JD, Echternach JL, Nof L, Gagnon Blodgett M: Test-retest reliability and minimal detectable change scores for the timed 'up \& go' test, the six-minute walk test, and gait speed in people with Alzheimer disease. Phys Ther 2009;89:569-579.

154 Ahlskog JE, Geda YE, Graff-Radford NR, Petersen RC: Physical exercise as a preventive or diseasemodifying treatment of dementia and brain aging. Mayo Clin Proc 2011;86:876-884. 